\author{
UNIVERSIDADE DE SÃO PAULO \\ MUSEU DE ARQUEOLOGIA E ETNOLOGIA \\ PROGRAMA DE PÓS-GRADUAÇÃO EM ARQUEOLOGIA
}

\title{
CONHECER PARA PRESERVAR: ARQUEOLOGIA E INCLUSÃO SOCIAL NA BACIA DO PARANAPANEMA SUPERIOR
}

Silvio Alberto Camargo Araújo

Tese apresentada ao Programa de PósGraduação em Arqueologia do Museu de Arqueologia e Etnologia da Universidade de São Paulo para a obtenção do título de Doutor.

Orientador: Profo ${ }^{\circ}$ Dr. José Luiz de Morais

Linha de Pesquisa: Gestão do Patrimônio Arqueológico

SÃO PAULO

2011 


\author{
UNIVERSIDADE DE SÃO PAULO \\ MUSEU DE ARQUEOLOGIA E ETNOLOGIA
}

\title{
CONHECER PARA PRESERVAR: ARQUEOLOGIA E INCLUSÃO SOCIAL NA BACIA DO PARANAPANEMA SUPERIOR
}

(VOLUME I)

Silvio Alberto Camargo Araújo 
Dedico este trabalho a minha mãe Maria Eunice por sua força, meus avôs José e Júnia pela criação e amor. Obrigado. 


\section{Agradecimentos}

Agradeço a Deus sobre todas as coisas que tenho na vida, família saúde, saúde, trabalho, amigos e colegas.

Ao Professor José Luiz de Morais pela orientação, respeito e confiança.

Aos Professores Maria Cristina de Oliveira Bruno, Camilo de Mello Vasconcelos, Marisa Coutinho Afonso, Raquel Glezer, Nestor Goulart Reis Filho, Sonia Hatsue Tatumi e Levy Figuti pela docência ou valiosa colaboração.

Aos técnicos do MAE/USP João Carlos, Paulinho e Dária.

Aos secretários e diretores municipais de cultura, agentes culturais, professores, informantes e colecionadores dos municípios visitados: Ana Paula Veiga Fogaça, Noemi Correa, Maria Rosa Pontes de Oliveira, Dona Rosinha, Ivanilda Prado, Sandra Mara Soares, Cristina Beatriz da Cruz, Lucilei da Silva Siqueira, Eni Brizola, Marieni Ângela Zilocchi Miguel, Priscila Held, Renata Fernandes, Simone Bonfim, Jaquelina Eli Leite Arruda, Davidson Panis Kaseker, Natanael Pires Lopes, Padre Djalma, João Bosco de Sá, Rui Furllani, José Maria Barros, Gilberto Cortes, Sebastião Pereira da Costa, Jandir A. Gonzaga, Preto Mattos, Helio Porto, Professor Laércio, Tenente Wagner Luciano de Oliveira, Paulo Raimundo de Freitas, Mário Bueno Sampaio, Milton Couto, Emmanuel Sócrates, João Luiz R. Ubaldo, Gilberto Lebre, Iolanda Guimarães, Sr Joaquim (Bilo), Maria Tereza Ferreira, Baltazar Preto, Denis Carvalho, José Passarinho e José Donato.

Aos Colaboradores: Kelly M. Scura de Oliveira, Marcio Lima, Emiliano Hagge, Alexandre Pereira de Souza, Renato Valério Rezende, Silvia Corrêa Marques, Olivia Piedade, Ernesto Magatti, Dr. Marcelo Nunes, Luciane Miwa Kamaze, família Butzer, família Braatz Moura, aos integrantes do Instituto Cílios da Terra - ICT, Instituto Histórico, Geográfico e Genealógico de Itapeva em especial professora Maria Olinda, Carlão, Wilson e aos funcionários da Prefeitura Municipal de Itapeva.

Aos historiadores que bravamente contribuíram no século $X X$ para manter viva a cultura e a historia regional: Cicero Marques, Genésio De Moura Müzel, Euflávio Barbosa, Leonor Ribeiro de Oliveira, Newton de Moura Müzel, João Dias Tatit e Eunice Brito Tatit. 
Aos donos das propriedades onde se situam os sítios estudados: Sra. Zina Fracarolli, Eduardo Fernando Fabri, Erineu Rodrigues da Silveira, Sr. Hugo Córdoba Ramos, em Sengés PR a família Rodrigues da Silva, pela cortesia e prestatividade com a qual fui recebido.

Aos meus familiares Samuel Augusto Camargo Araujo, Fernanda Kiomi Fontes Ferreira Camargo, José Carlos Moura Camargo todo apoio e cuidado dispensados a minha pessoa e filhos.

Aos meus filhos e sobrinhos me desculpem pelo tempo na frente do computador Eric, Thainá, Francine, Cesar, Marquinho, Allana, Arthur, Anderson, Rafael e Miguel, agradeço a paciência. 
Precisamos realmente de um público informado; não podemos nos dar o luxo de ficar isolados [...] Um público interessado e informado não destruirá seu próprio passado (nossa matéria prima).

Philip Rahtz 


\section{RESUMO}

Este trabalho apresenta a reunião de dados e informações sobre a arqueologia de 11 municípios do Paranapanema superior, lado paulista (municípios de Bonsucesso de Itararé, Buri, Capão Bonito, Guapiara, Itaberá, Itapeva, Itararé, Nova Campina, Ribeirão Branco, Ribeirão Grande e Taquarivai), os quais juntos tem uma população de mais de 250 mil habitantes e passaram nos últimos 50 anos por diversas pesquisas acadêmicas e preventivas que renderam fragmentos e artefatos de sítios pré-históricos e históricos, além de milhares de páginas de teses, dissertações e relatórios técnicos escritos. Existem também artefatos, sítios arqueológicos, coleções particulares e museus municipais que estão à margem das ações de salvaguarda, preservação, conservação e extroversão da arqueoinformação, que necessitam de fundamentos básicos. Sendo assim integrou-se a arqueoinformação dos diversos setores sociais envolvidos com o objetivo de preservar e potencializar o uso do patrimônio arqueológico e suas conjunções. Para tanto se buscou informações com lideranças políticas, sociais, memorialistas, professores das redes públicas de ensino, colecionadores, assim como a compreensão da legislação municipal e a utilização social que envolve o patrimônio arqueológico regional. Foi constatado em alguns casos o intenso uso do patrimônio arqueológico e histórico-arquitetônico e a intenção na preservação, mas todos os municípios se mostram carentes de informações técnicas e científicas enquanto outros municípios se mostram totalmente alheios aos bens que portam ou portavam. Como parte dos resultados obtidos na pesquisa construiuse alguns cenários de ocupações humanas para a área de estudo, consolidando-os em mapas, fotos e textos. Há necessidade da aproximação entre os segmentos sociais envolvidos (prefeituras, universidades, arqueólogos, museólogos, educadores, IPHAN, ONGs entre outros). No final se chega à conclusão de que não só é possível, mas muito importante para 0 desenvolvimento humano e ambiental da área de estudo (em especial a educação, a cultura e o turismo) a implantação de um Núcleo de Regional de Arqueologia e Meio Ambiente em Itapeva-SP que contribua, organize e sistematize por meio do conhecimento técnico científico o uso e a preservação do patrimônio arqueológico dos 11 municípios que se mostrou de considerável extensão.

Palavras-chave: arqueologia brasileira; Paranapanema superior; inclusão social do patrimônio arqueológico; cenários de ocupação humana. 


\section{ABSTRACT}

This paper presents a collection of data and information on the archaeology of 11 towns in the Upper Paranapanema, in the paulista region (Bonsucesso de Itararé, Buri, Capão Bonito, Guapiara, Itaberá, Itapeva, Itararé, Nova Campina, Ribeirão Branco, Ribeirão Grande and Taquarivaí) which, together, comprise a population of over 250,000 inhabitants and which, in the last 50 years, have been the focus of several preventive and academic researches, which yielded fragments and artifacts from prehistoric and historic sites, besides thousands of pages of theses, dissertations and recorded technical reports. There are also artifacts, archaeological sites, private collections and municipal museums which are on the fringe of the salvage, preservation and conservation policies. Feeding the archaeoinformation back on a regional level requires some basic principles. Hence, the archaeoinformation from several social sectors involved have been integrated with the goal to preserve and stimulate the use of the archaeological heritage and its connections. To accomplish that, political and social leaders, memorialists, public school teachers and collectors were sought. The aim was to understand the municipal legislation and the social use involved in the regional archaeological heritage. It was noticed, in some cases, the intensive use of the archaeological and historical heritage and an intention towards preservation, but all towns show a lack of technical and scientific information, while other places proved to be completely unaware of the assets they have or used to have. Some models of human occupation for the areas in context were developed with part of the results obtained, consolidating them on maps and illustrated texts. It is evident the need to bring together the social segments involved (municipalities, universities, archaeologists, museologists, educators, IPHAN (The National Historical and Artistic Heritage Institute), NGOs, among others). The conclusion is that it is not only possible but very important to the human development of the areas studied (especially education, culture and tourism) the establishment of a centre of regional archaeology to contribute to, organize and systematize through the application of technical and scientific knowledge the use and the preservation of the considerable archaeological heritage of the 11 towns investigated.

Keywords: brazilian archeology; upper Paranapanema; inclusion of archeology heritage; scenarios of human occupation. 


\section{SUMÁRIO}

INTRODUÇÃO

CAPÍTULO I: LOGÍSTICA DA PESQUISA

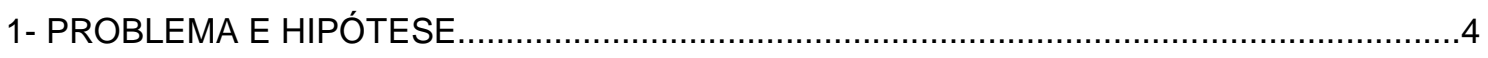

2- OBJETIVOS.

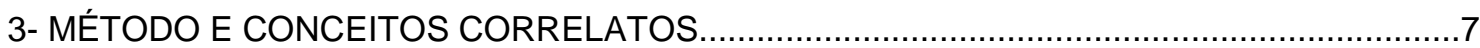

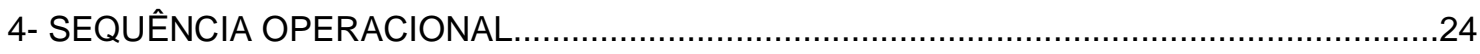

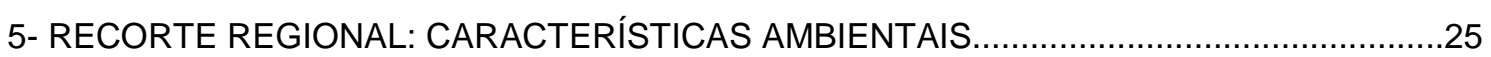

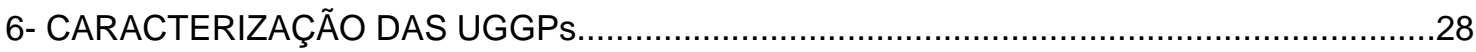

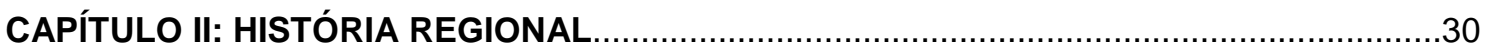

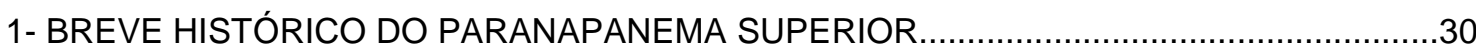

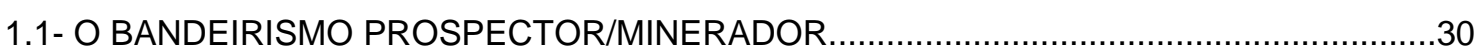

1.2- BANDEIRISMO DE APRESAMENTO

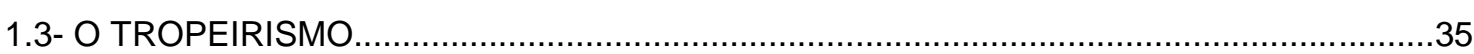

1.4- ALGODÂO, SUÍNOS E A AGROINDÚSTRIA INCIPIENTE..............................................43

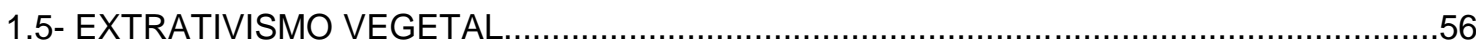

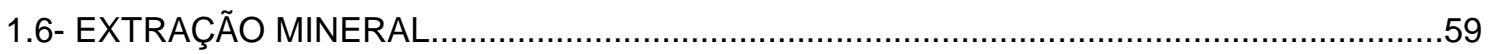

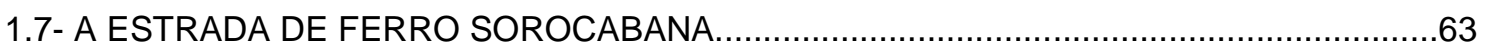

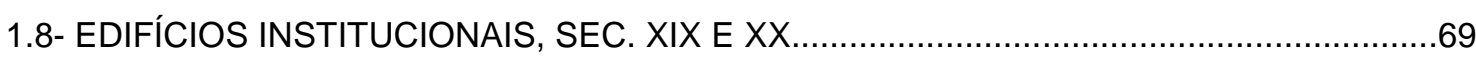

1.9- REVOLUÇÔES DE 1930/1932 E A MINERAÇÃO.............................................................

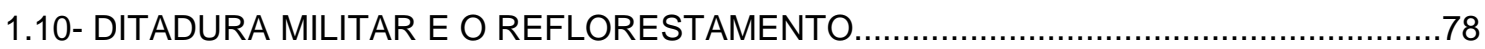

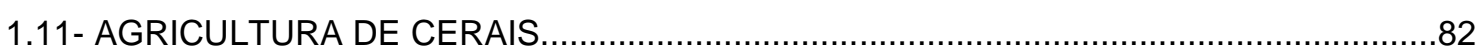

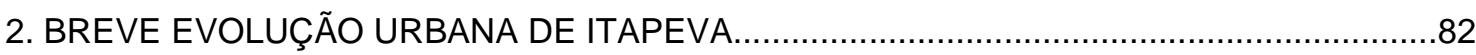

CÁPITULO III - ARQUEOLOGIA REGIONAL

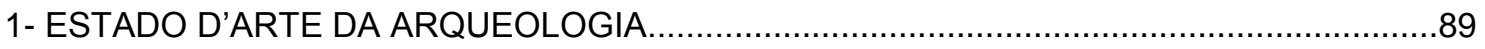

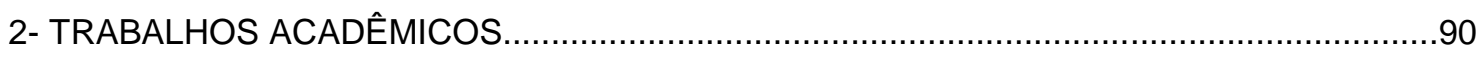

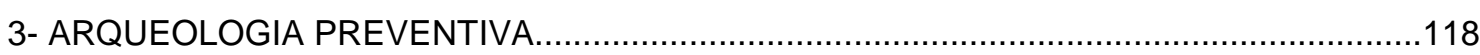

4-ARQUEOLOGIA PREVENTIVA NAS PROXIMIDADES DA ÀREA DE

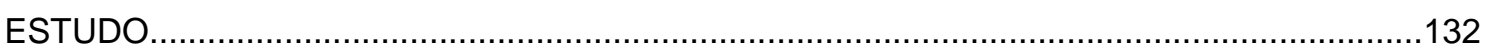

CAPÍTULO IV: ARQUEOINFORMAÇÂO E A ATUAL MUSEALIZAÇÃO ..............................134

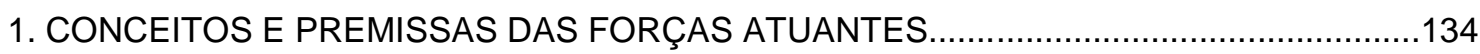

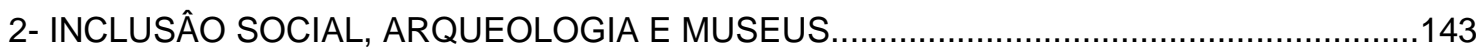

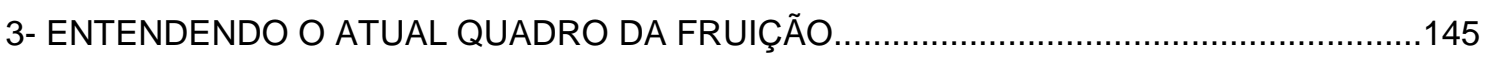

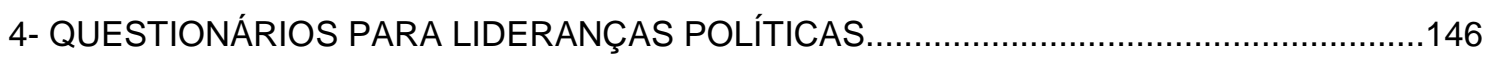


4.1- QUESTIONÁRIO PARA LIDERANÇAS POLÍTICAS: MUNICÌPIO DE BONSUCESSO DE ITARARÉ.

4.2- QUESTIONÁRIOS PARA LIDERANÇAS POLÍTICAS: MUNICìPIO DE

BURI. 151

4.3- QUESTIONÁRIOS PARA LIDERANÇAS POLÍTICAS: MUNICÍPIO DE CAPÃO BONITO

4.4- QUESTIONÁRIOS PARA LIDERANÇAS POLÍTICAS: MUNICìPIO DE

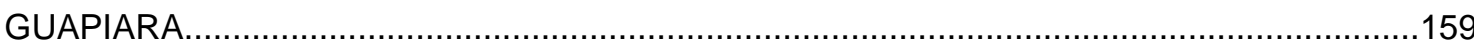

4.5- QUESTIONÁRIOS PARA LIDERANÇAS POLÍTICAS: MUNICÌPIO DE ITABERÁ. 161

4.6- QUESTIONÁRIOS PARA LIDERANÇAS POLÍTICAS: MUNICİPIO DE ITAPEVA 162

4.7- QUESTIONÁRIOS PARA LIDERANÇAS POLÍTICAS: MUNICÌPIO DE ITARARÉ 196

4.8- QUESTIONÁRIO PARA LIDERANÇAS POLÍTICAS: MUNICÌPIO DE NOVA CAMPINA 206

4.9- QUESTIONÁRIO PARA LIDERANÇAS POLÍTICAS: MUNICÌPIO DE RIBEIRÃO BRANCO.

4.10- QUESTIONÁRIOS PARA LIDERANÇAS POLÍTICAS: MUNICÌPIO DE RIBEIRÃO GRANDE. .212

4.11- QUESTIONÁRIO PARA LIDERANÇAS POLÍTICAS: MUNICÌPIO DE

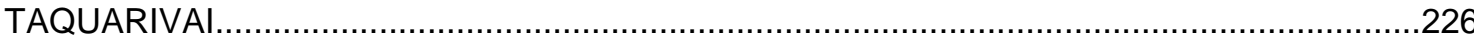

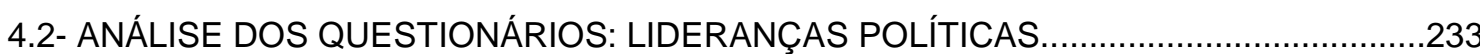

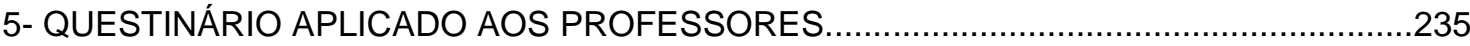

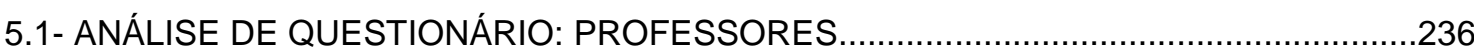

CAPÍTULO V: UM ENCONTRO FORTUÍTO NO CENTRO DE ITAPEVA.............................240

1- O SÍTIO HCR

CAPÍTULO VI: CONSOLIDAÇÂO DOS CENÁRIOS

1- CENÁRIO DE MACROSSISTEMA CAÇADORES-COLETORES (PALEOÍNDIO, CAÇADORCOLETOR ANTIGO E CAÇADOR-COLETOR RECENTE).

2- CENÁRIO DE MACROSSISTEMA DE AGRICULTORES (GUARANI, KAINGANG E TUPI)

3- CENÁRIO DE CONTATO INTERÉTNICO (APRESAMENTO DO ÍNDIO, BUSCA AO OURO

E TROPEIRISMO).

4- FORTALECIMENTO DA AGROPECUÀRIA E A EFS. 284

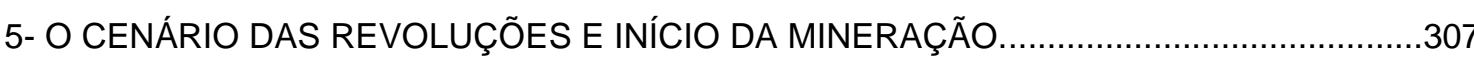

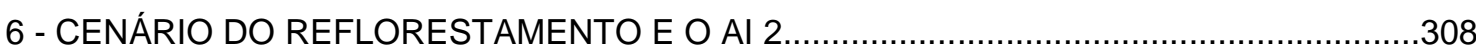

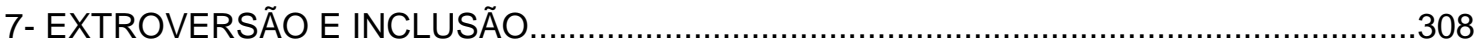


CAPITTULO VII: CONCLUSÃO

BIBLIOGRAFIA. 
LISTA DE FOTOS

FOTO 1: BARRACÃO DE BENEFICIAMENTO DE ALGODÃO EM ITAPEVA NO ANO DE 1921

FOTO 2: BARRACÃO DE BENEFICIAMENTO DE ALGODÃO EM ITAPEVA DA DÉCADA DE 1920 EM USO EM 2011

FOTO 3: BARACÃO DE BENEFICIAMENTO DE ALGODÃO CONSTRUÍDO EM 1918 PERTENCENTE A LUÍS CARRIEL. ESTADO DE USO E CONSERVAÇÃO EM 2011. VISTA FRONTAL

FOTO 4: BARACÃO DE BENEFICIAMENTO DE ALGODÃO CONSTRUÍDO EM 1918 PERTENCENTE A LUÍS CARRIEL. ESTADO DE USO E CONSERVAÇÃO EM 2011. VISTA DOS FUNDOS

FOTO 5: BARRACÃO DE BENEFICIAMENTO DE ALGODÃO DA SANBRA. S.A. EM 2011. ATUAL ESTADO DE USO E CONSERVAÇÂO.

FOTO 6: TRANSPORTE DE ALGODÃO EM ITAPEVA DA FAXINA, DÉCADA DE 1920.

FOTO 7: TRANSPORTE DE ALGODÃO EM ITAPEVA DA FAXINA, DÉCADA DE 1920 (LOCAL NÃO IDENTIFICADO)

FOTO 8: TRNSPORTE DE ALGODÃO NO RIO DAS ALMAS EM CAPÃO BONITO NA DÉCADA DE 1920.

FOTO 9: ADELINO ROLIM EM MEIO A PLANTAÇÃO DE ALGODÂO NA DÉCADA DE 1930. FAZENDA PILÃO D'ÁGUA.

FOTO 10: DEPÓSITO DE PORCOS EM ITARARÉ NA DÉCADA DE 1920. . .53

FOTO 11: BARRACÂO DA AGROINDÚSTRIA DE ANTÔNIO PELISSARI EM 2011. VISTA EXTERNA FRONTAL.

FOTO 12: BARRACÂO DA AGROINDÚSTRIA DE ANTÔNIO PELISSARI EM 2011. VISTA INTERNA

FOTO 13: CASA DE DEFUMAÇÃO DA ANTIGA AGROINDÚSTRIA DE ANTÔNIO PELISSARI EM 2011

FOTO 14: ANTIGA MANGUEIRA DE PORCOS DA AGROINDÚSTRIA DE ANTÔNIO PELISSARI EM 2011

FOTO 15: CURTUME “ROBERTO BUTZER E GEHRING” EM 1930 .....................................55

FOTO 16: CURTUME “ROBERTO BUTZER E GEHRING” EM 2011 .......................................55

FOTO 17: VISTA INTERNA DO CURTUME "ROBERTO BUTZER E GEHRING" EM 2011.

FOTO 18: CALDEIRA DO CURTUME "ROBERTO BUTZER E GEHRING" EM 2011

FOTO 19: SGUARIO MADEIRAS NA DÉCADA DE 1940 .58

FOTO 20: SERRARIA DO CERRADO EM CAPÃO BONITO NA DÉCADA DE 1920 .58

FOTO 21: SERRARIA EM BURI NAS MARGNES DO RIO APIAÍ NA DÉCADA DE 1920. 
FOTO 22: VISTA FRONTAL DA COMPANHIA DE CIMENTO PORTLAND MARINGÁ NA DÉCADA DE 1950

FOTO 23: VISTA DA VILA OPERÀRIA DA COMPANHIA DE CIMENTO PORTLAND MARINGÁ NA DÉCADA DE 1950

FOTO 24: VISTA DA ECOSTA COM CAIEIRAS DE JAMIL LEÃO BRASILEIRO NO ANO DE 2011.

FOTO 25: VISTA DE FORNO PARA CAL DE JAMIL LEÃO BRASILEIRO CONTRUÍDO NA DÉCADA DE 1940.

FOTO 26: VISTA DA INSERÇÃO NA PAISAGEM URBANA DOS FORNOS DE JAMIL LEÃO BRASILEIRO EM 2011

FOTO 27: FORNOS DE CIMENTO PARA PRODUÇÂO DE CAL DA FAZENDA POUSO ALTO E BORDA NO ANO DE 2011 .

FOTO 28: OFICINA E VILA OPERÁRIA DA FAZENDA POUSO ALTO E BORDA NO ANO DE 2011

FOTO 29: DETALHE DE FORNO DE CIMENTO, VISTA OBLÍQUA .62

FOTO 30: DETALHE DA BOCA DE FORNO DE CIMENTO, VISTA FRONTAL. .62

FOTO 31: DETALHE DOS FORNOS DE TIJOLOS PARA A PRODUÇÃO DE CAL. FAZENDA POUSO ALTO E BORDA NO ANO DE 2011.

FOTO 32: DETALHE DA BOCA DO FORNO DE TIJOLOS PARA A PRODUÇÃO DE CAL. FAZENDA POUSO ALTO E BORDA NO ANO DE 2011.

FOTO 33: ESTAÇÂO FERROVIÁRIA DE ITARARÉ NA DÉCADA DE 1920.............................65

FOTO 34: ESTAÇÂO FERROVIÁRIA DE FAXINA NA DÉCADA DE 1920..............................65

FOTO 35: ESTAÇÂO FERROVIÁRIA DE NA DÉCADA DE 1920 VISTA A PARTIR DA VILA OPERÀRIA.

FOTO 36: PONTE DA ESTRADA DE FERRO SOROCABANA NAS PROXIMIDADES DE FAXINA.......

FOTO 37: ESTAÇÂO FERROVIÁRIA DE ENGENHEIRO MAIA (s/d)...................................67

FOTO 38: ESTAÇÂO FERROVIÁRIA DO JAÓ EM 1909...................................................67

FOTO 39: ESTAÇÂO FERROVIÁRIA ENGENHEIRO BACELLAR EM 1909...........................67

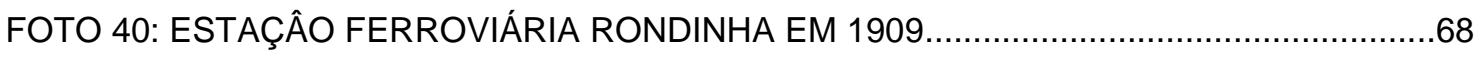

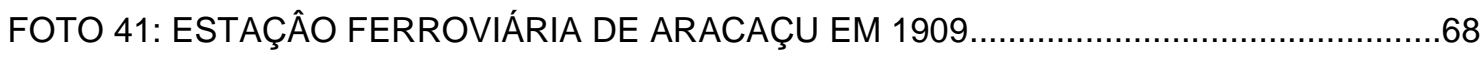

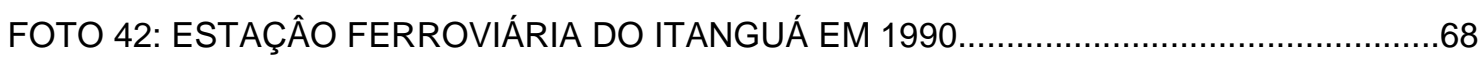

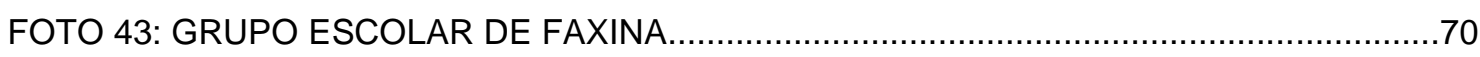

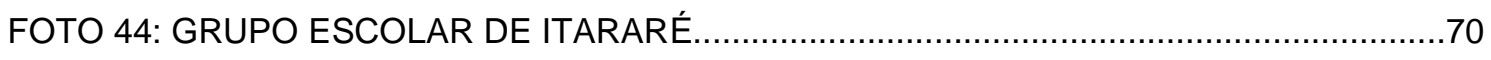

FOTO 45: GRUPO ESCOLAR DE CAPÃO BONITO .........................................................

FOTO 46: CÂMARA MUNICIPAL DE FAXINA (S/D) .................................................... 


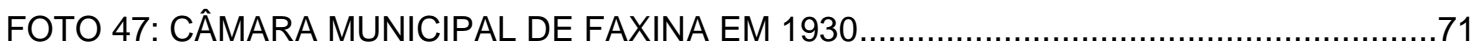

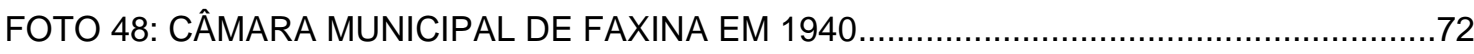

FOTO 49: SANTA CASA DE MISERICÓRDIA POR VOLTA DE 1920......................................72

FOTO 50: SANTA CASA DE MISERICÓRDIA NA DÉCADA DE 1950 ....................................72

FOTO 51: SANTA CASA DE MISERICÓRDIA NA DÉCADA DE 1950 COM TAIPA AO

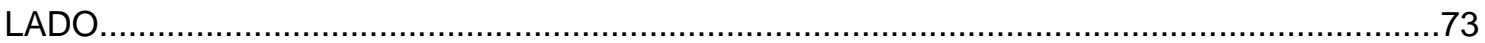

FOTO 52: SANTA CASA DE MISERICÓRDIA DE ITAPEVA NA DÉCADA DE 1980................73

FOTO 53: GABINETE DE LEITURA DE FAXINA NA DÉCADA DE 1920...............................73

FOTO 54: GRUPO ESCOLAR FAXINA/DOM SILVIO MARIA DÁRIO......................................74

FOTO 55: SEMINARIO PROPEDÊUTICO NA DÉCADA DE 1990...........................................74

FOTO 56: ESTAÇÃO CORONEL IZALTINO EM SENGÉS NO PARANÁ.................................76

FOTO 57: REVOLUCIONÁRIOS ENTRINCHEIRADOS AO REDOR DE ITARARÉ...................76

FOTO 58; GETÚLIO VARGAS EM ITARARÉ EM 28 DE OUTUBRO DE 1930.........................77

FOTO 59: BATALHÃO UNIVERSITÁRIO PAULISTA EM FOTO DE ESTÚDIO..........................77

FOTO 60: GENERAL CASTELO BRANCO E PREFEITO ANTÔNIO CAVANI...........................80

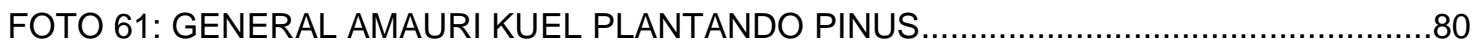

FOTO 62: GENERAL CASTELO BRANCO PLANTADO PAU-BRASIL...................................81

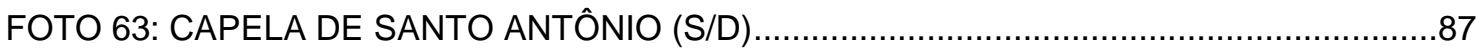

FOTO 64: CAPELA DE SANTO ANTÔNIO EM 1949 ANTES DE SER DEMOLIDA...................87

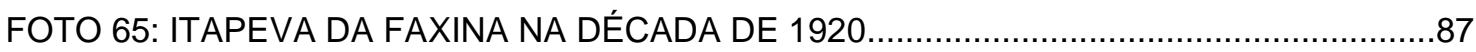

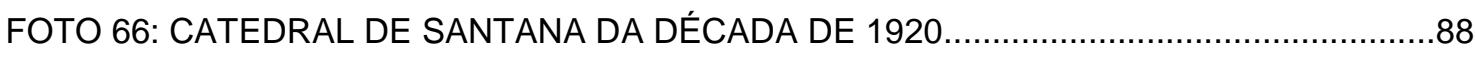

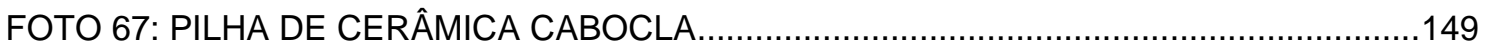

FOTO 68: PROCESSO ARTESANAL DE PRODUÇÃO CERÂMICA.....................................150

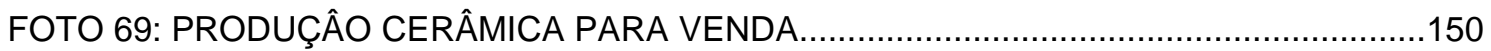

FOTO 70: ESTAÇÃO FERROVIÁRIA DE BURI - FRENTE ..............................................152

FOTO 71: ESTAÇÃO FERROVIÁRIA DE BURI - FUNDO COM PLATAFORMA.....................152

FOTO 72: ESTAÇÃO FERROVIÁRIA DE BURI, VISTA INTERNA COM ARTESÃS................152

FOTO 73: MUNICÃO ATRIBUIDA A REVOLUÇÃO DE 1932 EXPOSTAS EM BURI................152

FOTO 74: AGENTE LOCAL EM TRINCHEIRA DA REVOLUÇÃO DE 1932...........................153

FOTO 75: EDIFÍCIO DA DÉCADA DE 1920 EM BURI. VISTA FRENTE/LATERAL.................153 
FOTO 76: EDIFÍCIO DA DÉCADA DE 1920 EM BURI. VISTA FRONTAL

FOTO 77: CANTIL.

FOTO 78: BAIONETAS.

FOTO 79: PISTOLA LLAMA. .157

FOTO 80: GRANADA DE MÃO..

FOTO 81: MUNIÇÕES E CÁPSULAS DE VÁRIOS CALIBRES .157

FOTO 82: ESTRUTURA METÁLICA DE FUZIL MODELO 1908. 158

FOTO 83: ESCOLA JACYRA LANDIM STORI FRENTE. 158

FOTO 84: ESCOLA JACYRA LANDIM STORI FUNDOS. 158

FOTO 85: CASARÃO COM CARACTERÍSTICAS COLONIAIS EM CAPÃO BONITO. .159

FOTO 86: TRICHEIRA ATRIBUÍDA A REVOLUÇÃO DE 1932. 161

FOTO 87: CASAMATA ATRIBUÍDA A REVOLUÇÃO DE 1932. 161

FOTO 88: PAIOL COM TELHAS COLONIAIS. 161

FOTO 89: TRENÓ PARA BALDEAR LENHA. .161

FOTO 90: FRENTE LATERAL DA ESTAÇÃO CULTURA... 167

FOTO 91: FUNDOS DA ESTAÇÃO CULTURA .167

FOTO 92: RESERVA TÉCNICA DA ESTAÇÃO CULTURA. 167

FOTO 93: MÓVEIS ANTIGOS DA RESERVA TÉCNICA. . .168

FOTO 94: ARTEFATOS DA REVOLUÇÃO DE 1932. 168

FOTO 95: PRATELEIRA COM TELAS E TELEFONES ANTIGOS .168

FOTO 96: ARTEFATOS DE USO DOMÉSTICO DO FINAL DO SÉCULO XIX E INÍCIO DO SÉCULO XX. 168

FOTO 97: INSTRUMENTO MUSICAL E MATERIAL DE ESCRITÓRIO. 168

FOTO 98: CÂMERAS FOTOGÁFICAS ANTIGAS 168

FOTO 99: MÁQUINAS DE ESCREVER ANTIGAS E RÁDIOS. 169

FOTO 100: OBJETOS VARIADOS DA ESTAÇÃO CULTURA (RELÓGIO, FÓSSEIS, CERÂMICAS)... 169

FOTO 101: VASILHA CERÂMICA DE 1920. 169

FOTO 102: LOUÇAS DO INÍCIO DO SÉCULO XX. 169

FOTO 103: VISTA PARCIAL DOS FUNDOS DA CASA DA CULTURA CÍCERO MAREQUES 
FOTO 104: VISTA PARCIAL DOS FUNDOS DA CASA DA CULTURA CÍCERO MARQUES.

FOTO 105: VISTA DA FACHADA DA CASA DA CULTURA CÍCERO MARQUES EM

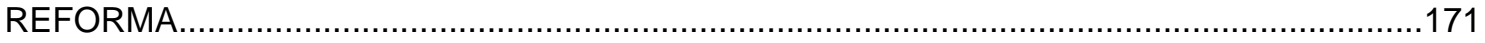

FOTO 106: ALICERCE DA CASA DA CULTURA COM TELHAS IDEAL/ORIGINAL...............171

FOTO 107: ALICERCE DA CASA DA CULTURA DSCARACTERIZADO...............................171

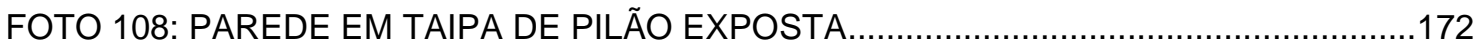

FOTO 109: TRINCA NA PAREDE NOS FUNDOS DA CASA DA CULTURA CÍCERO MARQUES.

FOTO 110: CASA GRANDE DA FAZENDA PILÃO D'ÁGUA POR VOLTA DE 1920...............173

FOTO 111: CONSTRUÇÃO DE BARRAGEM DA REPRESA DO PILÃO D'ÁGUA...................173

FOTO 112: CONSTRUÇÃO DE BARRAGEM DA REPRESA DO PILÃO D'ÁGUA...................173

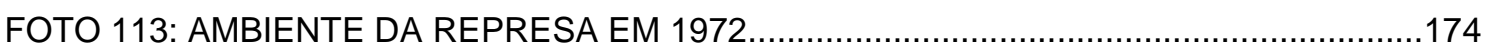

FOTO 114: ILHA ARTIFICIAL DA REPRESA DO PILÃO D'ÁGUA EM

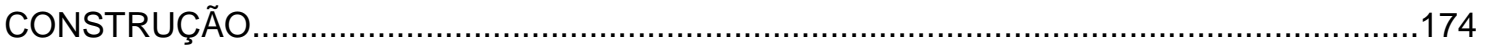

FOTO 115: ALICERCE DE RESTAURANTE EM CONSTRUÇÃO.........................................174

FOTO 116: AMBIENTE DA REPRESA DO PILÃO D'ÁGUA VISTO DA RODOVIA SP-258 EM

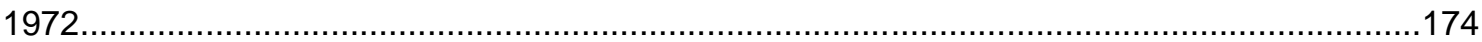

FOTO 117: RECANTO BENTO ALVES NATEL POUCO ANTES DA INAUGURAÇÃO EM

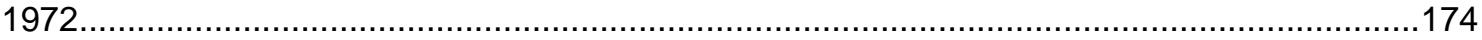

FOTO 118: RECANTO BENTO ALVES NATEL EM 20 DE SETEMBRO DE

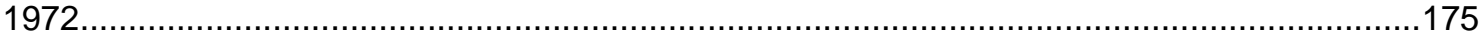

FOTO 119: CASA GRANDE DA FAZENDA PILÃO D'ÁGUA................................................178

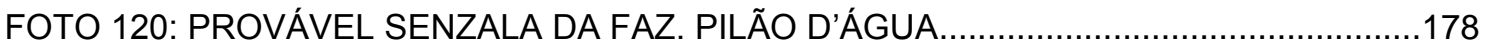

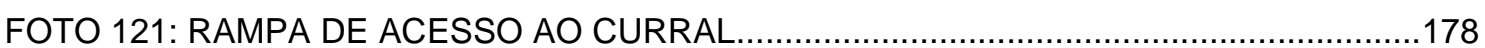

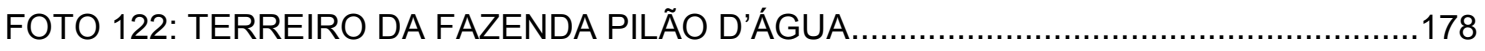

FOTO 123: VISTA FRONTAL DA PROVÁVEL SENZALA FAZENDA PILÃO

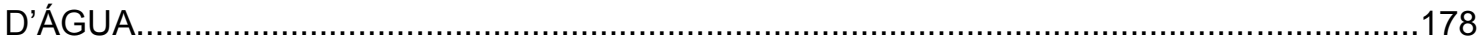

FOTO 124: VISTA EM PERSPECTIVA DA PROVÁVEL SENZALA FAZENDA PILÃO

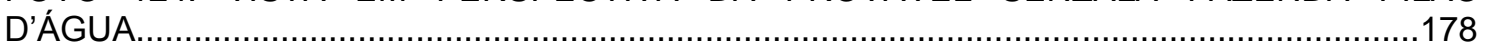

FOTO 125: ESTRUTURAS DE PEDRAS DA FEZENDA PILÃO D’ÁGUA EM 2005................179

FOTO 126: ESTRUTURAS DE PEDRAS DA FEZENDA PILÃO D'ÁGUA EM 2005................179

FOTO 127: LOCALIZAÇÃO DAS ANTIGAS ESTRUTURAS DE

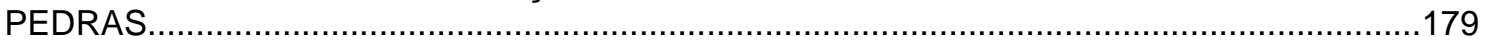

FOTO 128: LOCALIZAÇÃO DAS ANTIGAS ESTRUTURAS DE PEDRAS...............................179 
FOTO 129: ATERRO PRÓXIMO AS ESTRUTURAS DE PEDRAS DENOMINADAS MUROS

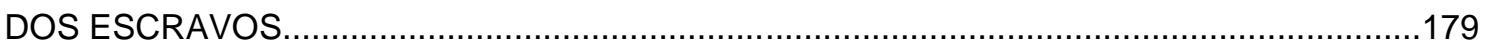

FOTO 130: BLOCOS DE ROCHAS REMOVIDOS PRÓXIMO AO MURO LESTE....................179

FOTO 131: MURO DE PEDRAS DESTRUÍDO POR ABERTURA DE ESTRADA, MURO NORTE.....

FOTO 132: MURO DE PEDRAS DESTRUÍDO POR ABERTURA DE ESTRADA, MURO NORTE.

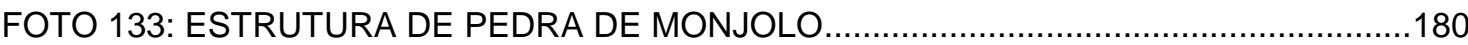

FOTO 134: ALICERCE DE ESTRUTURA DE PEDRAS DO MONJOLO ..................................180

FOTO 135: VISTA FRONTAL DA CAPELA DA FAZENDA PILÃO D'ÁGUA...........................181

FOTO 136: VISTA FRENTE/LATERAL DA CAPELA DA FAZENDA PILÃO D’ÁGUA...............181

FOTO 137: CASA GRANDE DA FAZENDA PILÃO D’ÁGUA NO ANO DE $2011 \ldots \ldots \ldots \ldots \ldots \ldots . . . . . . . .181$

FOTO 138: VISTA FRONTAL DA CASA GRANDE DO PILÃO D’ÁGUA................................181

FOTO 139: NÚCLEO DE SOLO ANTROPOGÊNICO EM FRENTE À CASA GRANDE DA

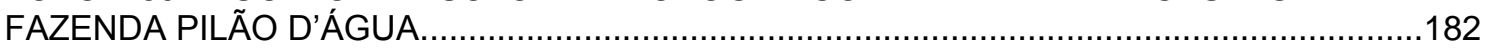

FOTO 140: FRAGMENTO DE CERÂMICA CABOCLA EM FRENTE A CASA GARNDE DA

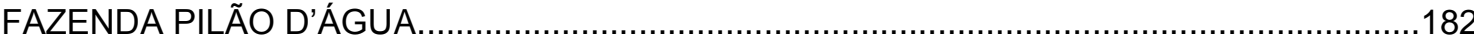

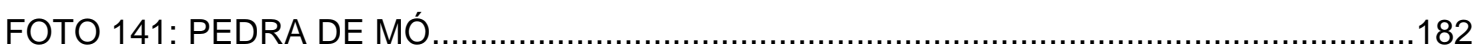

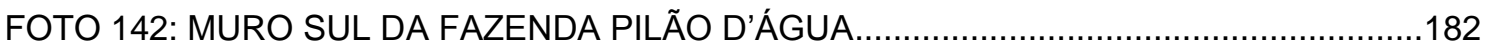

FOTO 143: FUNDOS DA CASA GRANDE DA FAZ. PILÃO D'ÁGUA ……...........................182

FOTO 144: EDIFÍCIOS DAS OFICINAS DE CERÂMICA E MARCENARIA.............................182

FOTO 145: EXPOSIÇÃO DE ARTEFATOS ARQUEOLÓGICOS INDÍGENAS........................183

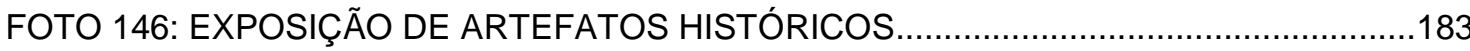

FOTO 147: TIGELA CERÂMICA DO INÍCIO DO SÉCULO XX..............................................183

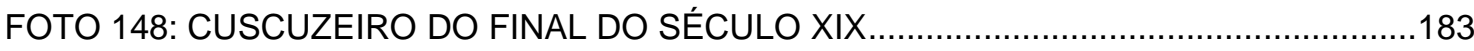

FOTO 149: CUSCUZEIRO DO FINAL DO SÉCULO XIX COM TRATAMENTO INADEQUADO

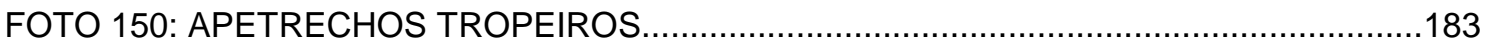

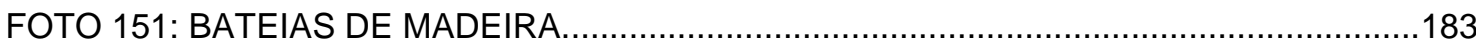

FOTO 152: VISTA PARCIAL DO RECANTO JORGE ASSUMPÇÃO SCHIMIDT......................184

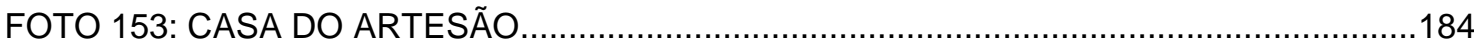

FOTO 154: VISTA PARCIAL DO RECANTO JORGE ASSUMPÇÃO SCHIMIDT E REPRESA

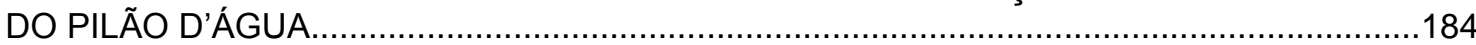

FOTO 155: CERÂMICA DE ARTESÃOS DE BONSUCESSO DE ITARARÉ............................184 


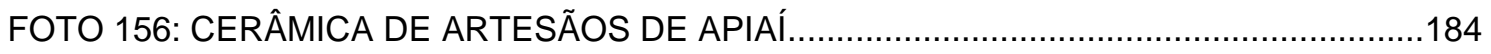

FOTO 157: ESTÁTUAS DE CERÂMICA DE ARTESÃOS DE ITAPEVA..................................185

FOTO 158: ESTÁTUAS DE CERÂMICA DE ARTESÃOS DE ITAPEVA.................................185

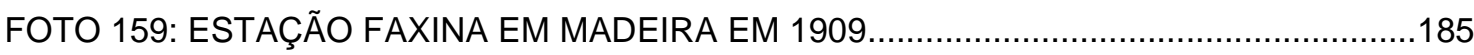

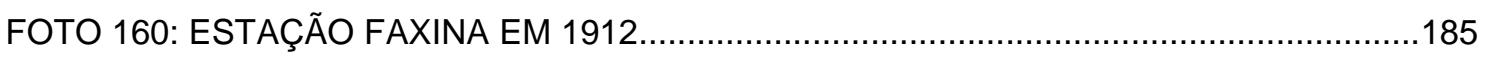

FOTO 161: ESTAÇÃO FAXINA EM 1912, VISTA LATERAL A PARTIR DOS TRILHOS..........186

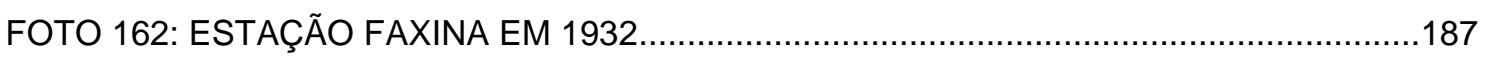

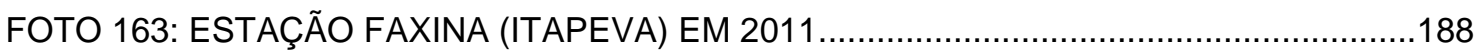

FOTO 164: VISTA DA TORRE DA ESTAÇÃO FAXINA (ITAPEVA) EM 2011 ........................188

FOTO 165: VISTA DO SAGUÃO DA ESTAÇÃO FAXINA (ITAPEVA) EM 2011 ......................188

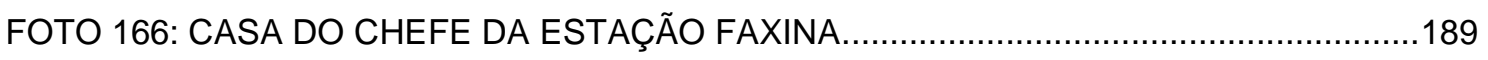

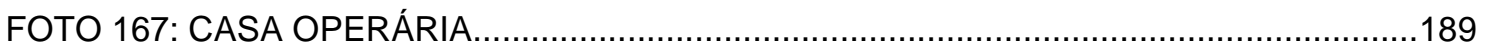

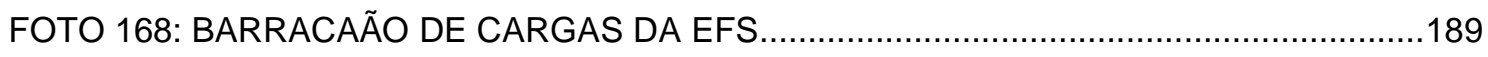

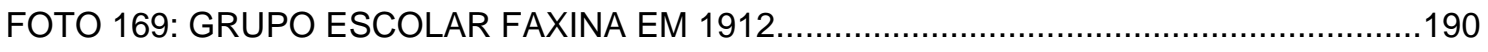

FOTO 170: ESCOLA ACÁCIO PIEDADE, EX- GRUPO ESCOLAR FAXINA EM 2011 ............190

FOTO 171: ANTIGO FÓRUM DESEMBARGADOR EUCLIDES DE CAMPOS NA DÉCADA DE

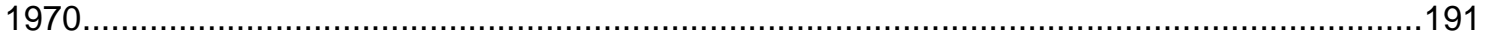

FOTO 172: ANTIGO FÓRUM DESEMBARGADOR EUCLIDES DE CAMPOS NA DÉCADA DE

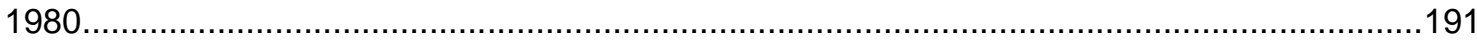

FOTO 173: ANTIGA CÂMARA DE VEREADORES E CADEIA PÚBLICA, 54 BATALHÃO DA POLÍCIA MILITAR NO ANO DE 2010 .....................................................................192

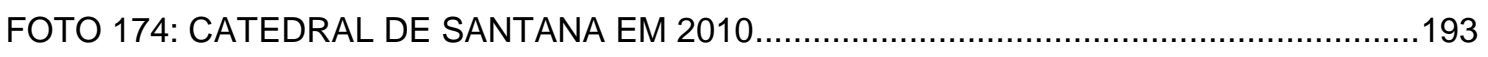

FOTO 175: CATEDRAL DE SANTANA NA DÉCADA DE 1980_...........................................193

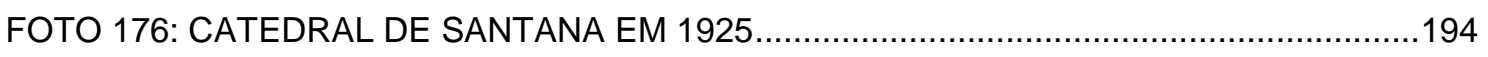

FOTO 177: CATEDRAL DE SANTANA EM 1910_.........................................................194

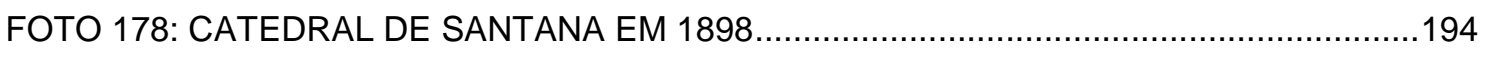

FOTO 179: CAPELA DO CARMO POR VOLTA DA DÉCADA DE 1920.................................195

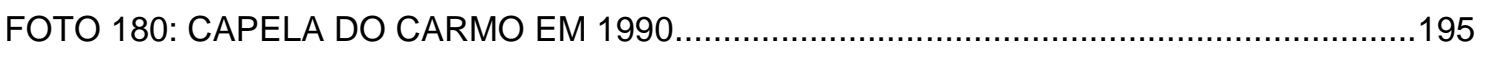

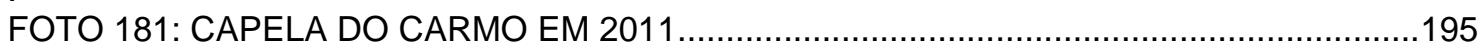

FOTO 182: SEMINÁRIO PROPEDÊUTICO NA DÉCADA DE 1980......................................196

FOTO 183: SEMINÁRIO PROPEDÊUTICO EM 1994............................................................196 
FOTO 184: VISTA PARCIAL DE MUSEU EM ITARARÉ.

FOTO 185: FOTOS EXPOSTAS DO MUSEU DE ITARARÉ

FOTO 186: EXPOSIÇÃO DE ARTEFATOS ARQUEOLÓGIOS INDÍGENAS NO MUSEU DE ITARARÉ.

FOTO 187: EXPOSIÇÃO DE ARTEFATOS HISTÓRICOS (ESTRIBOS) NO MUSEU DE ITARAÉ.

FOTO 188: CÁPSULAS DE ARTILHARIA RELACIONADAS AS REVOLUÇÕES DE 1930/1932 EXPOSTAS NO MUSEU DE ITARARÉ.

FOTO 189: VISTA PARCIAL DA ANTIGA SALA QUE ABRIGA VA O MUSEU DE ITARARÉ EM 2008. 198

FOTO 190: VISTA FRONTAL DA ESTAÇÃO FERROVIÁRIA DE ITARARÉ. 199

FOTO 191: VISTA DOS FUNDOS DA ESTAÇÃO FERROVIÁRIA DE ITARARÉ. 199

FOTO 192: VISTA FRENTE/LATERAL DA ESTAÇÃO FERROVIÁRIA DE ITARARÉ (EFS). 199

FOTO 193: SOBRADO DA EFS DA ESTAÇÃO FERROVIÁRIA DE ITARARÉ........................200

FOTO 194: SOBRADO DA RVPSC DA ESTAÇÃO FERROVIÁRIA DE ITARARÉ..................200

FOTO 195: DETALHE FRENTE/LATERAL DA ESTAÇÃO FERROVIÁRIA DE

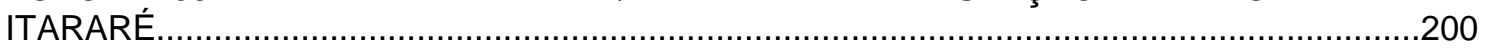

FOTO 196: DETALHE FRENTE/LATERAL DA ESTAÇÃO FERROVIÁRIA DE

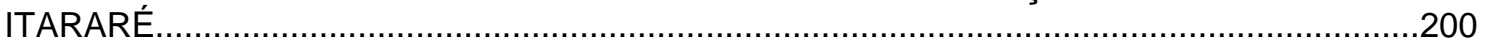

FOTO 197: MÁQUINA FOTOGRÁFICA DE CLARO G. JANSSON......................................202

FOTO 198: MATERIAL FOTOGRÁFICO DE CLARO G. JANSON.........................................202

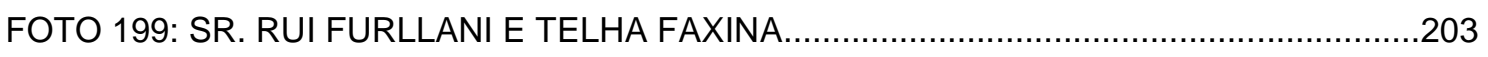

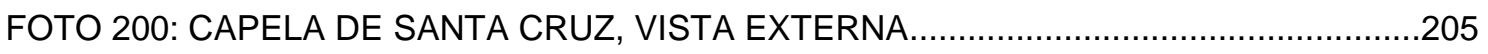

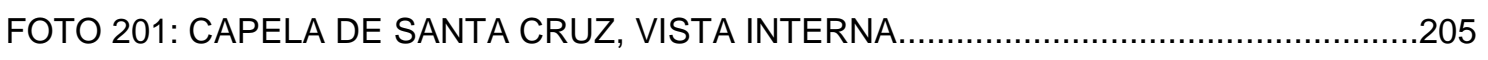

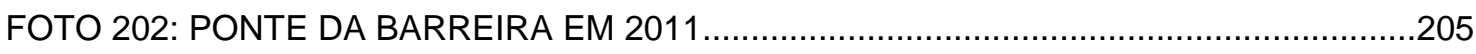

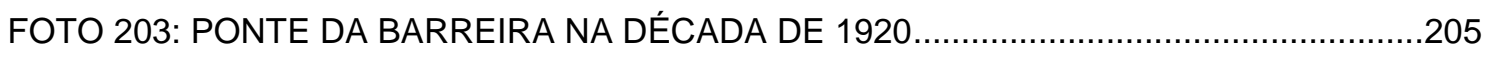

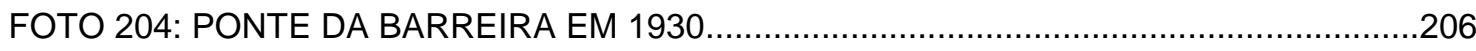

FOTO 205: ESTROMATÓLITOS DE ITAPEVA E NOVA CAMPINA........................................209

FOTO 206: ESTROMATÓLITOS DE ITAPEVA E NOVA CAMPINA.......................................209

FOTO 207: ESTROMATÓLITOS DE ITAPEVA E NOVA CAMPINA.......................................209

FOTO 208: ESTROMATÓLITOS DE ITAPEVA E NOVA CAMPINA.......................................209

FOTO 209: COLEÇÃO DE ARTEFATOS ARQUEOLÓGICOS DA FAMÍLIA BRAATZ MOURA....... 
FOTO 210: COLEÇÃO DE ARTEFATOS ARQUEOLÓGICOS DA FAMÍLIA BRAATZ MOURA

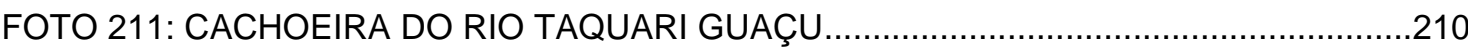

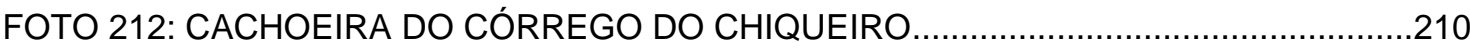

FOTO 213: CASAS ANTIGAS NO CENTRO DE RIBEIRÃO BRANCO .................................212

FOTO 214: CASAS ANTIGAS NO CENTRO DE RIBEIRÃO BRANCO..................................212

FOTO 215: ARTEFATOS DE COLECIONADORES DE RIBEIRÃO GRANDE .......................214

FOTO 216: ARTEFATOS DE COLECIONADORES DE RIBEIRÃO GRANDE........................214

FOTO 217: ARTEFATOS DE COLECIONADORES DE RIBEIRÃO GRANDE .......................214

FOTO 218: ARTEFATOS DE COLECIONADORES DE RIBEIRÃO GRANDE........................214

FOTO 219: VISTA FRONTAL DA CASA GRANDE DE RIBEIRÃO GRANDE.........................215

FOTO 220: VISTA DOS FUNDOS DA CASA GRANDE DE RIBEIRÃO GRANDE..................216

FOTO 221: VISTA INTERNA DA CASA GRANDE DE RIB. GRANDE..................................216

FOTO 222: DETALHE DO TELHADO DA CASA GRANDE DE RIBEIRÃO GRANDE.............216

FOTO 223: FRAGMENTO DE TELHA COLONIAL NA RAÍZ DE UMA PITANGUEIRA...........216

FOTO 224: FRAGMENTOS DE TELHAS COLONIAIS NO FUNDO DO LOTE DA CASA

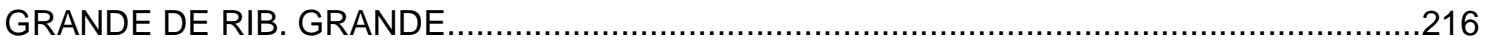

FOTO 225: EXPOSIĈÃO DE ARTEFATOS ARQUEOLÓGICOS DENTRE OUTROS - CASA

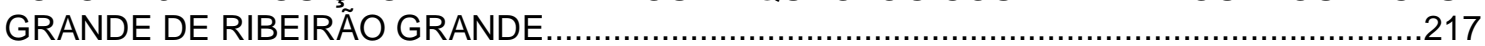

FOTO 226: ARTEFATOS DE CERÃMICA E MADEIRA EXPOSTAS NA CASA GRANDE DE RIB. GRANDE

FOTO 227: ARTEFATOS CERÂMICOS EXPOSTOS NA CASA GRANDE DE RIB. GRANDE

FOTO 228: TRABALHOS EXOLARES EXPOSTOS NO INTERIOR DA CASA

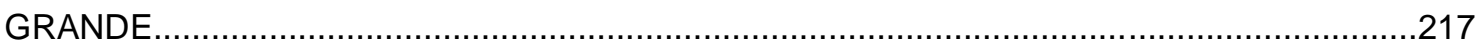

FOTO 229: PANELAS DE FERRO E TORRADOR DE FARINHA.........................................217

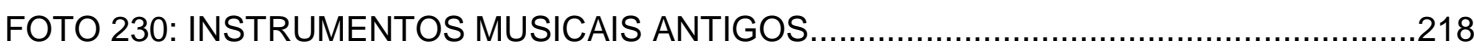

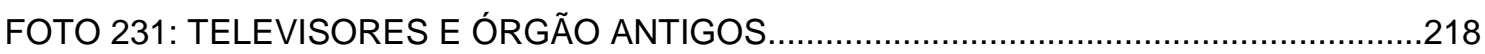

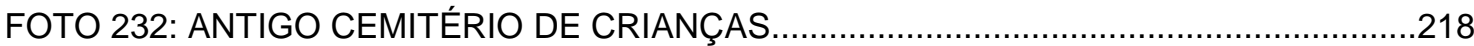

FOTO 233: CASA COM TELHAS COLONIAIS NO CENTRO DE RIBEIRÃO GRANDE.........218.

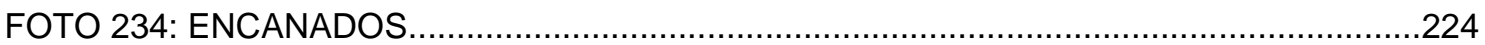

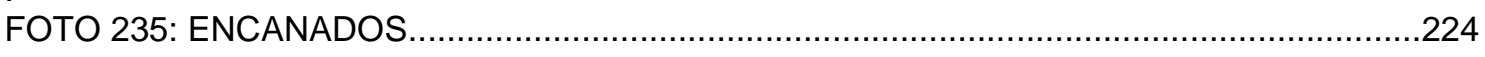

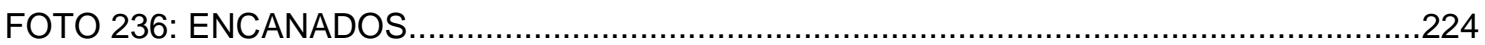


FOTO 237: ENCANADOS.

FOTO 238: ENCANADO TIPO ILHA FLUVIAL..

FOTO 239: PERFIL LATERAL ESQUERDO DE ENCANADO ILHA FLUVIAL. .225

FOTO 240: PERFIL CENTRAL DE ENCANADO ILHA FLUVIAL. .225

FOTO 241: PERFIL LATERAL DIREITO DE ENCANADO ILHA FLUVIAL. .225

FOTO 242: CAPELA DE VILA VELHA NA DÉCADA DE 1950 .228

FOTO 243: CAPELA DE VILA VELHA NA DÉCADA DE 1970. 228

FOTO 244: CAPELADE VILA VELHA EM 2011 .229

FOTO 245: INTERIOR DA CAPELA DE VILA VELHA. 229

FOTO 246: VISTA DO FUNDO DA CAPELA DE VILA VELHA. .229

FOTO 247: VISTA DA CAPELA DE VILA VELHA E CEMITÉRIO. .229

FOTO 248: DETALHE DO ALTAR DA CAPELA DE VILA VELHA. .230

FOTO 249: DETALHE DO ALTAR DA CAPELA DE VILA VELHA. .230

FOTO 250: VISTA DA COLUNA INTERNA DA CAPELA DE VILA VELHA. .230

FOTO 251: DETALHE DA COLUNA INTERNA DA CAPELA DE VILA VELHA.. .230

FOTO 252: TELHAS COLONIAIS SOB AS RAÍZES DE UM JERIVÁ. .230

FOTO 253: PAIOL REAPROVEITANDO TELHAS TIPO COLONIAL .230 FOTO 254: FUNDO DA CASA ADJACENTE A CAPELA E CEMITÉRIO DE VILA VELHA ....231 FOTO 255: FRENTE CASA ADJACENTE A CAPELA E CEMITÉRIO DE VILA VELHA..........231 FOTO 256: CAPELA SENHORA CONCEIÇÃO CENTRO DE TAQUARIVAÍ............................232 FOTO 257: DETALHE DA CAPELA SENHORA CONCEIÇÃO.............................................232 FOTO 258: DETALHE DA CAPELA SENHORA CONCEIÇÃO............................................232 FOTO 259: VISTA INTERNA DA CAPELA SENHORA CONCEIÇÃO....................................233 FOTO 260: VISTA INTERNA DA CAPELA SENHORA CONCEIÇÃO....................................233

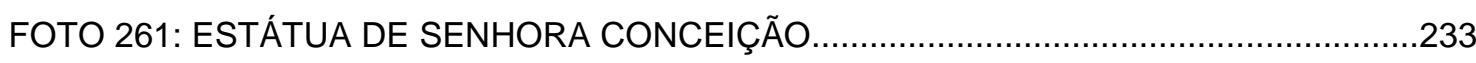

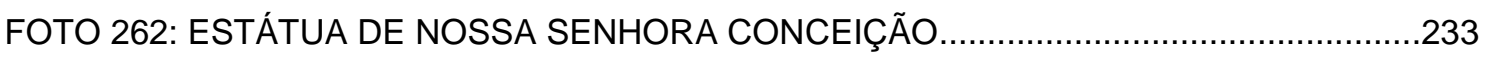
FOTO 263: ESTRUTURA ARQUEOLÓGICA SOB OS ESCOMBROS - CONJUNTO.............246 FOTO 264: ESTRUTURA ARQUEOLÓGICA SOB OS ESCOMBROS - OSSOS E CERÂMICAS....... 246

FOTO 265: CASARÃO SANTA FÉ NA DÉCADA DE 1990 .247 FOTO 266: CASARÃO SANTA FÉ DEMOLIDO - NO ALICERCE. .247 
FOTO 267: CASARÃO SANTA FÉ DEMOLIDO - NO ALICERCE E CASA VIZINHA, FUTURO SÍTIO HCR.

FOTO 268: PAREDE DE TAIPA DO CASARÃO SANTA FÉ EM DEMOLIÇÃO 247

FOTO 269: PAREDE DE TAIPA DO CASARÃO SANTA FÉ - DETALHE DE RIPAS DE JERIVÁ 247

FOTO 270: CASARÃO SANTA FÉ. .248

FOTO 271: SÍTIO HCR 248

FOTO 272: SONDAGEM COM NSA 248

FOTO 273: SONDAGEM. 248

FOTO 274: SONDAGEM. 248

FOTO 275: PERFIL DE BARRANCO LADO SUL, ALICERCE. 248

FOTO 276: PERFIL DE BARRANCO LADO NORTE COM NSA 248

FOTO 277: VISTA PARCIAL DOS LOTES UNIFICADOS - SÍTIO HCR. 249

FOTO 278: TRINCHEIRA 1 EVIDENCIANDO ESTRUTURA DE LIXO - SÍTIO HCR 249

FOTO 279: TRINCHEIRA 1 COM ESTRUTURA DE LIXO - SÍTIO HCR 249

FOTO 280: LÍTICO LASCADO DE CAÇADOR COLETOR DE RIBEIRÃO GRANDE 257

FOTO 281: PONTAS DE PROJÉTEIS DE COLECIONADOR EM RIBEIRÃO GRANDE 257

FOTO 282: PONTAS DE PROJÉTEIS E PRÉ FORMAS DA COLEÇÃO DA SECRETARIA MUNICIPAL DE CULTURA E TURISMO DE ITAPEVA. 258

FOTO 283: PONTA BIFACIAL DE QUARTZO LEITOSO DO ALTO TAQUARI 258

FOTO 284: UNIFÁCIE DE QUARTZITO DO ALTO TAQUARI .258

FOTO 285: ARTEFATOS LÍTICOS DO ALTO TAQUARI. 258

FOTO 286: LítICOS BUMERANGÓIDE DE SÍLEX E RASPADOR DE ARENITO SILICIFICADO. 258

FOTO 287: URNA LISA GUARANI SÍTIO FONSECA. 265

FOTO 288: URNA CORRUGADA SÍTIO CAÇADOR 265

FOTO 289: URNA CORRUGADA E TAMPA GUARANI DO SITIO FONSECA. 265

FOTO 290: URNAS GUARANI FRAGMENTADAS SÍTIO SILVEIRA. 265

FOTO 291: URNA CORRUGADA GUIARANI DO ACERVO DA SECRETARIA MUNICIPAL DE CULTURA E TURISMO DE ITAPEVA. 266

FOTO 292: BORDUNA E MÃOS DE PÍLÃO DO ACERVO DA SECRETARIA MUNICIPAL DE CULTURA E TURISMO DE ITAPEVA. 266

FOTO 293: MACHADO POLIDO DO SR. PAULO SAPONGA .266 
FOTO 294: COLEÇÃO DE LÍTICOS POLIDOS DA SECRETARIA MUNICPAL DE CULTURA DE ITAPEVA. 266

FOTO 295: VASILHA ITARARÉ COM CARENA DO SR. JOSÉ

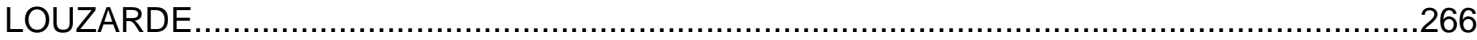

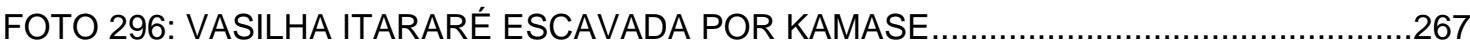

FOTO 297: PARTE DE VASILHA ITARARÉ ESCAVADA POR KAMASE ...............................267

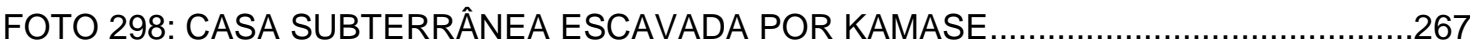

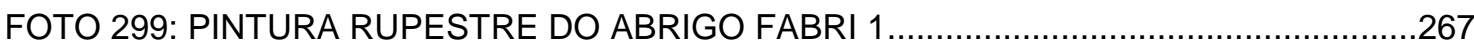

FOTO 300: PINTURA RUPESTRE DO ABRIGO FABRI 1 COM SEGREGAÇÃO DE

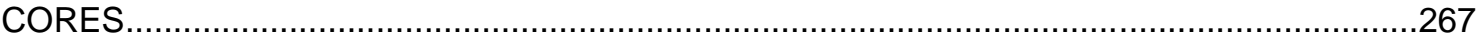

FOTO 301: PINTURA RUPESTRE DO ABRIGO FABRI 1 - CERVÍDEO................................268

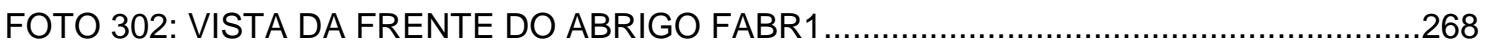

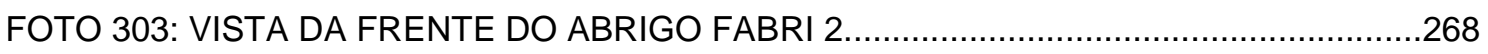

FOTO 304: CAMPO RUPESTRE DO ALTO DA SERRA DAS PEDRAS................................268

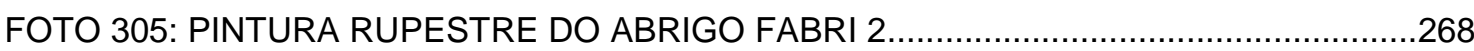

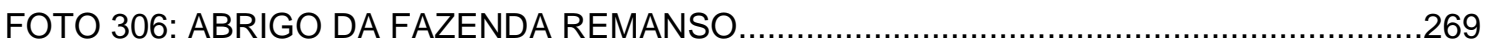

FOTO 307: PAINEL RUPESTRE DO ABRIGO ITAPEVA EM 211 ........................................26

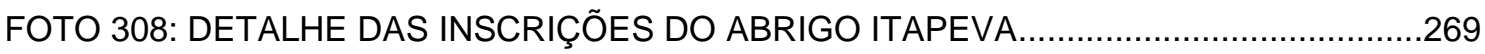

FOTO 309: DETALHE DAS INSCRIÇÕES DO ABRIGO ITAPEVA ........................................270

FOTO 310: DETALHE DAS INSCRIÇÕES DO ABRIGO ITAPEVA.......................................270

FOTO 311: PINTURAS RUPESTRES AO LADO DAS INSCRIÇÕES DO ABRIGO

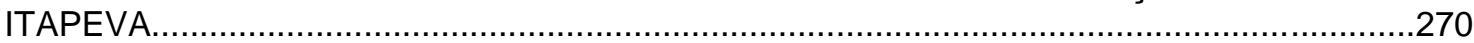

FOTO 312: PINTURAS RUPESTRES AO LADO DAS INSCRIÇÕES DO ABRIGO ITAPEVA

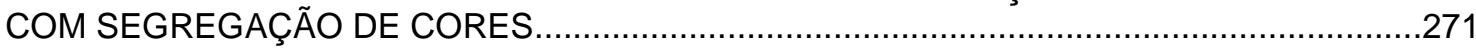

FOTO 313: INSERÇÃO NA PAISAGEM DO SCF-4

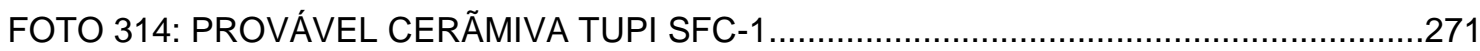

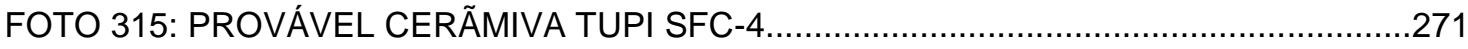

FOTO 316: ENCANADOS NO BAIRRO DOS ANACLETOS ...............................................274

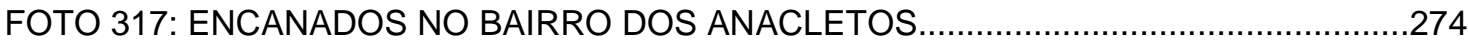

FOTO 318: CERÂMICA LISA MARROM FINA - SÍTIO SANTA MARIA ..................................275

FOTO 319: CERÂMICA COM ENGOBO BRANCO - SÍTIO SANTA MARIA............................275

FOTO 320: CHAVE DE METAL ANTIGA - SÍTIO SANTA MARIA.........................................275

FOTO 321: FRAGMENTO CERÂMICO CORRUGADO - SÍTIO SANTA MARIA......................275 
FOTO 322: FRAGMENTO CERÂMICO ESCOVADO COM ALÇA-SÍTIO SANTA

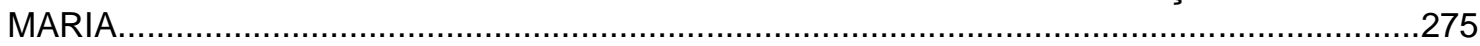

FOTO 323: FRAGMENTO CERÂMICO COM APLIQUE NA BORDA.....................................275

FOTO 324: VASO CORRUGADO, ROLETADO NA BORDA E COM INCISÕES DUPLAS.

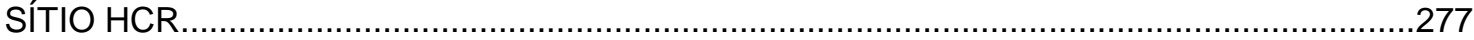

FOTO 325: RECONSTITUIÇÃO PARCIAL DE VASO CORRUGADO, FUNDO DA VASILHA SÍTIO HCR......

FOTO 326: RECONSTITUIÇÃO PARCIAL DE VASO CORRUGADO, PERFIL LATERAL SÍTIO HCR

FOTO 327: FRAGMENTO CERÂMICO CORRUGADO - SÍTIO HCR .278

FOTO 328: RECONSTITUIÇÃO PARCIAL DE VASILHA ESCOVADA/CARIMBADA - SÍTIO HCR......

FOTO 329: DETALHE DA RECONSTITUIÇÃO PARCIAL DE VASILHA

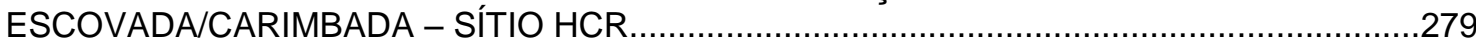

FOTO 330: RECONSTITUIÇÃO DE MORINGA CERÂMICA - SÍTIO HCR..............................279

FOTO 331: FRAGMENTO DE PEQUENA CERÂMICA LISA - SÍTIO HCR.............................279

FOTO 332: FRAGMENTO DE CERÂMICA UNGULADA/ESCOVADA - SÍTIO HCR

FOTO 333: PERFIL DE FRAGMENTO DE CERÂMICA UNGULADO/ESCOVADO - SÍTIO HCR...... 280

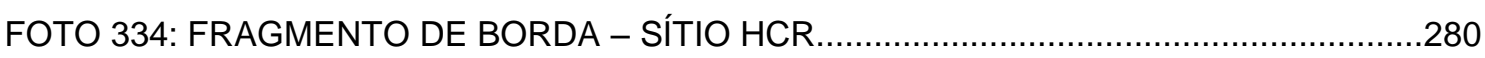

FOTO 335: PERFIL DE FRAGMENTO DE BORDA - SÍTIO HCR........................................280

FOTO 336: PERFIL DE FRAGMENTO DE PRATO CERÂMICO MARROM - SÍTIO HCR

FOTO 337: MURO DE PEDRAS SOBREPOSTAS COM ARGAMASSA DA FAZENDA MORUNGAVA.

FOTO 338: DETALHE DE MURO DE PEDRAS SOBREPOSTAS COM ARGAMASSA DA FAZENDA MORUNGAVA.

FOTO 339: CANTO DE MURO DE PEDRAS SOBREPOSTAS COM ARGAMASSA DA

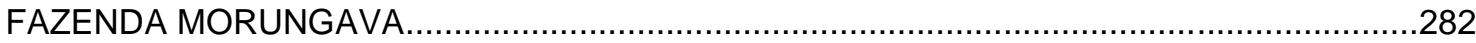

FOTO 340: MURO DE PEDRAS SOBREPOSTAS COM ARGAMASSA DA FAZENDA MORUNGAVA.

FOTO 341: ARTEFATOS ARQUEOLÓGICOS E HISTÓRICOS DA FAZENDA MORUNGAVA 282

FOTO 342: SR. ADELINO NO TERREIRO DA FAZENDA PILÃO D’ÁGUA NA DÉCADA DE 1930. 283

FOTO 343: TERREIRO DA FAZENDA PILÃO D’ÁGUA EM 2011. 284 


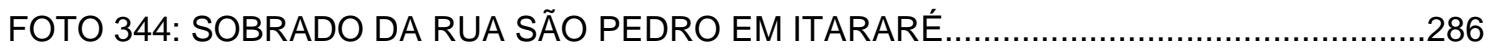

FOTO 345: CASARÃO DA PRAÇA CORONEL JORDÃO EM ITARARÉ.................................286

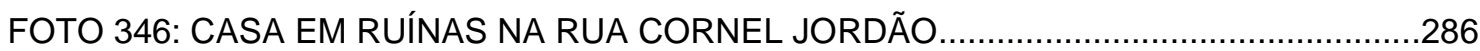

FOTO 347: CALÇADA DA RUA SÃO PEDRO

FOTO 348: RUÍNAS DA BENEFICIADORA DE ALGODÃO SANTA NÉLIA EM

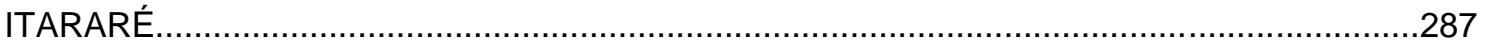

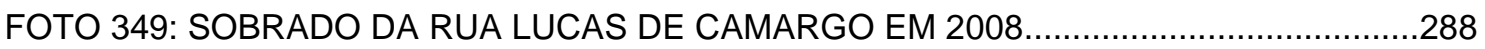

FOTO 350: SOBRADO DA RUA LUCAS DE CAMARGO DESCARACTERIZADO EM

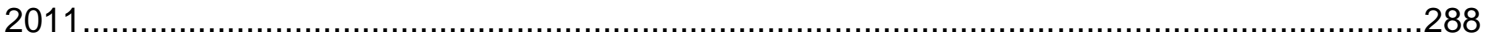

FOTO 351: EDIFÍCIO RESIDENCIAL DA PRAÇA ANCHIETA...............................................28

FOTO 352: ÁREA DO EDIFÍCIO RESIDENCIAL DA PRAÇA ANCHIETA ..............................289

FOTO 353: ESTAÇÃO FERROVIÁRIA ARACAÇU - BURI. VISTA A PARTIR DA VILA

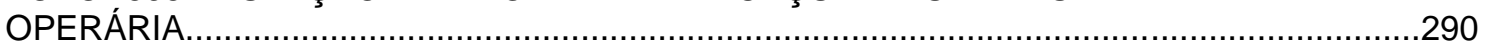

FOTO 354: ESTAÇÃO FERROVIÁRIA ARACAÇU - BURI. VISTA A PARTIR DO

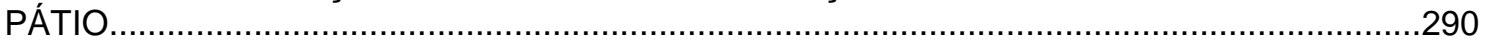

FOTO 355: VISTA LATERAL DA ESTAÇÃO FERROVIÁRIA ARACAÇU - BURI. LADO LESTE. .290

FOTO 356: VISTA LATERAL DA ESTAÇÃO FERROVIÁRIA ARACAÇU - BURI. LADO OESTE .290

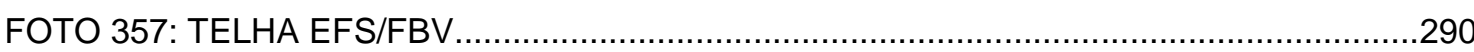

FOTO 358: DETALHE DO ALICERCE DA ESTAÇÃO ARACAÇU - BURI...............................290

FOTO 359: VISTA DO INTERIOR DA ESTAÇÃO ARACAÇU - BURI....................................291

FOTO 360: VISTA DO INTERIOR DA ESTAÇÃO ARACAÇU - BURI....................................291

FOTO 361: CASA DA (EFS) ESTAÇÃO FERROVIÁRIA ARACAÇU - BURI...........................291

FOTO 362: DETALHE DE VISTA FRONTAL DE CASA DA (EFS) ESTAÇÃO FERROVIÁRIA

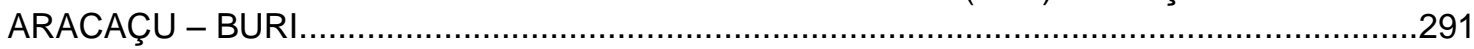

FOTO 363: DETALHE DE VISTA LATERAL DE CASA DA (EFS) ESTAÇÃO FERROVIÁRIA

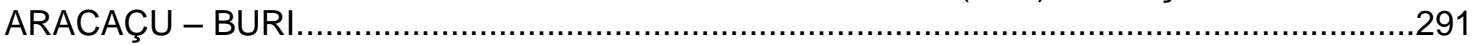

FOTO 364: CASAS DA VILA OPERÁRIA DA ESTAÇÃO FERROVIÁRIA DE ARACAÇU -

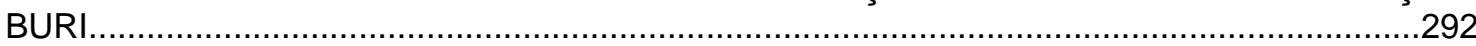

FOTO 365: CASAS DA VILA OPERÁRIA DA ESTAÇÃO FERROVIÁRIA DE ARACAÇU BURI.

FOTO 366: CASAS DA VILA OPERÁRIA DA ESTAÇÃO FERROVIÁRIA DE ARACAÇU BURI.......

FOTO 367: CASAS DA VILA OPERÁRIA DA ESTAÇÃO FERROVIÁRIA DE ARACAÇU BURI. 
FOTO 368: LOCAL DA ANTIGA ESTAÇÃO VITORINO CARMILO - VISTA GERAL

FOTO 369: LOCAL DA ANTIGA ESTAÇÃO VITORINO CARMILO - POÇO E TANQUE DE LAVAR ROUPAS.

FOTO 370: TIJOLO COM A SIGLA NSA 293

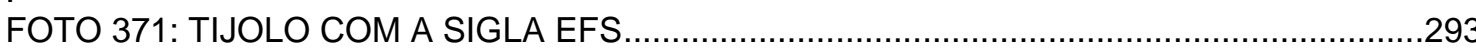

FOTO 372: ESTAÇÃO FERROVIÁRIA DE BURI - VISTA LATERAL.....................................293

FOTO 373: ALICERCE DA ESTAÇÃO FERROVIÁRIA DE BURI ENCOBERTO POR SEDIMENTOS

FOTO 374: PLACA E ESTAÇÃO FERROVIÁRIA DE BURI. 294

FOTO 375: DETALHE DA PLACA DA ESTAÇÃO FERROVIÁRIA DE BURI. 294

FOTO 376: LOCAL DA ANTIGA ESTAÇÃO FERROVIÁRIA RONDINHA EM

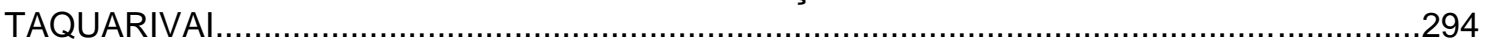

FOTO 377: LOCAL DA ANTIGA ESTAÇÃO FERROVIÁRIA RONDINHA EM

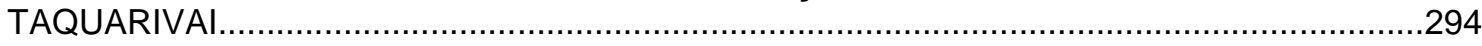

FOTO 378: ALICERCE DE CASA DA ESTAÇÃO FERROVIÁRIA

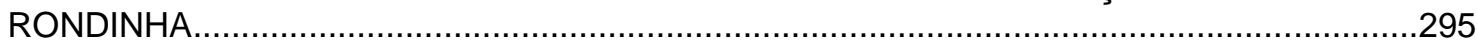

FOTO 379: VISTA PARCIAL DA OCUPAÇÃO DO SOLO DA ESTAÇÃO

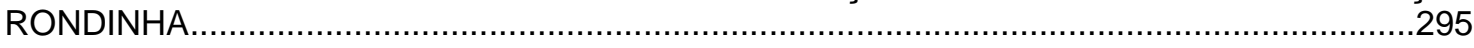

FOTO 380: RUÍNAS DA ESTAÇÃO FERROVIÁRIA ENG. BACELLAR ITAPEVA

FOTO 381: PLACA DA ESTAÇÃO FERROVIÁRIA ENG. BACELLAR - ITAPEVA..................296

FOTO 382: VISTA DAS CASAS OPERÁRIAS ESTAÇÃO FERROVIÁRIA ENG. BACELLAR -

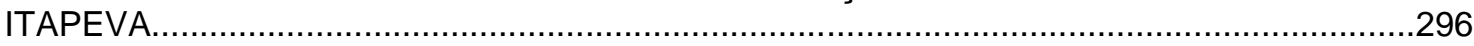

FOTO 383: TIJOLO J*B ENCONTRADO NA ESTAÇÃO FERROVIÁRIA ENG. BACELLAR ITAPEVA. .296

FOTO 384: TELHA SACOMAN FRERES/VILA PRUDENTE ENCONTRADA NA ESTAÇÃO

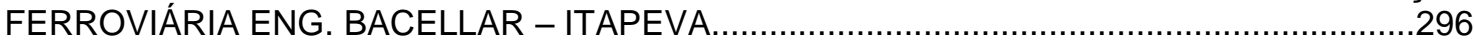

FOTO 385: ESTAÇÃO FERROVIÁRIA DO JAÓ - ITAPEVA...............................................297

FOTO 386: ALICERCE DE CASA DA ESTAÇÃO FERROVIÁRIA DO JAÓ...............................297

FOTO 387: ESTRUTURA DE TIJOLOS A VISTA DA ANTIGA CAIXA D'ÁGUA DA ESTAÇÃO

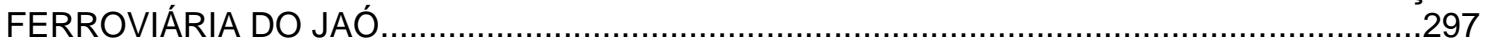

FOTO 388: TIJOLO TATUI - ESTAÇÃO FERROVIÁRIA DO JAÓ.........................................298

FOTO 389: TIJOLO EFS - ESTAÇÃO FERROVIÁRIA DO JAÓ.............................................298

FOTO 390: TIJOLO LORETI - ESTAÇÃO FERROVIÁRIA DO JAÓ........................................298

FOTO 391: TIJOLO DN - ESTAÇÃO FERROVIÁRIA DO JAÓ..................................................298 
FOTO 392: FERRAGEM PARA TRILHOS - ESTAÇÃO ALFREDO NUNES

FOTO 393: GARRAFA DE VIDRO - ESTAÇÃO ALFREDO NUNES......................................299

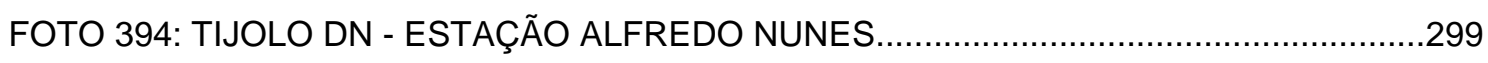

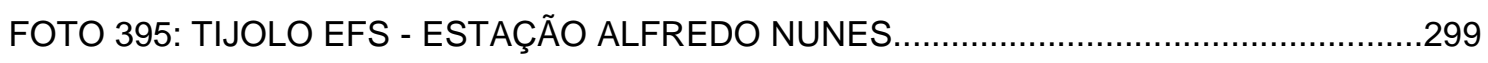

FOTO 396: TANQUE DE LAVAR ROUPAS - ESTAÇÃO ALFREDO NUNES..........................299

FOTO 397: ALICERCE DA ESTAÇÃO - ESTAÇÃO ALFREDO NUNES.................................299

FOTO 398: ESTAÇÃO FERROVIÁRIA DO ITANGUÁ - VISTA LESTE..................................300

FOTO 399: ESTAÇÃO FERROVIÁRIA DO ITANGUÁ - VISTA OESTE................................300

FOTO 400: ESTAÇÃO FERROVIÁRIA DO ITANGUÁ - VISTA LATERAL..............................300

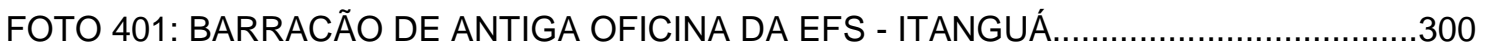

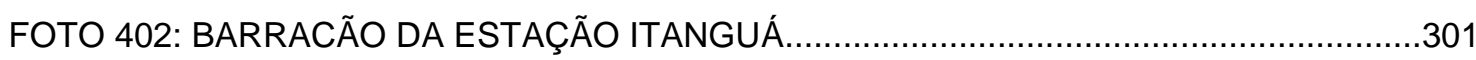

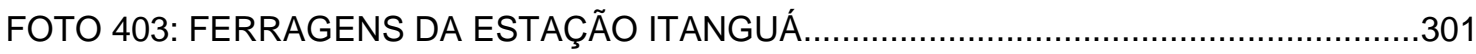

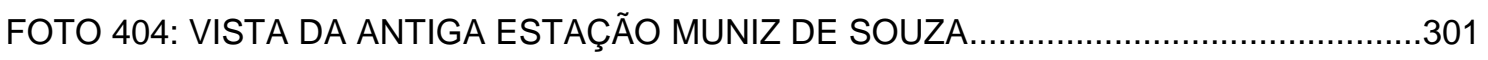

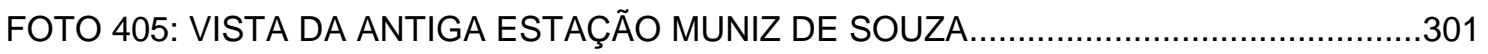

FOTO 406: CASA DE JOSÉ PASSARINHO - ESTAÇÃO ENG. MAIA...................................302

FOTO 407: CASA DO SR. NELCY OLIVEIRA - ESTAÇÃO ENG. MAIA...................................302

FOTO 408: VISTA DA PLATAFORMA EM RUÍNAS DA ESTAÇÃO ENG. MAIA......................302

FOTO 409: LOCAL DA ANTIGA ESTAÇÃO FERROVIÁRIA GORITA.....................................303

FOTO 410: VISTA PARCIAL DA PLATAFORMA DA ESTAÇÃO FERROVIÁRIA

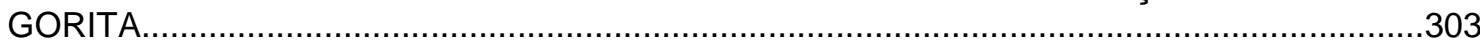

FOTO 411: ESTRUTURA DE TIJOLOS AVISTA DA ESTAÇÃO FERROVIÁRIA

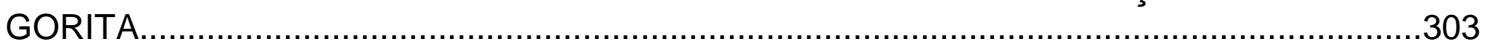

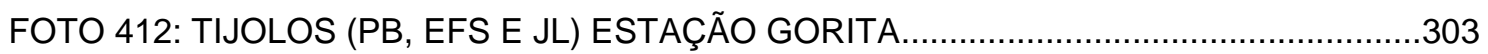

FOTO 413: VISTA PARCIAL DA ESTAÇÃO FERROVIÁRIA RIO VERDE..............................304

FOTO 414: ALICERCE DE CASA DA ESTAÇÃO RIO VERDE.............................................304

FOTO 415: RUÍNAS DA PLATAFORMA DA ESTAÇÃO FERROVIÁRIA RIO VERDE..............304

FOTO 416: CAIXA D’ÁGUA COMPLETA DA ESTAÇÃO FERROVIÁRIA RIO VERDE.............305

FOTO 417: DETALHE DA CAIXA D'ÁGUA DA ESTAÇÃO FERROVIÁRIA RIO VERDE

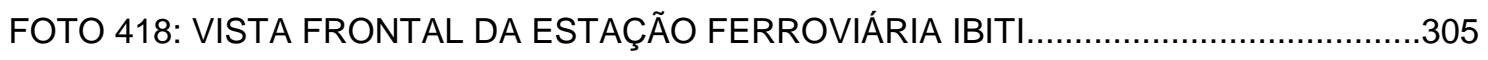

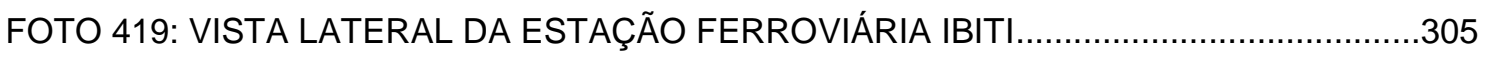

FOTO 420: BARRACÃO AO LADO DA ESTAÇÃO FERROVIÁRIA DE ITARARÉ...................307 
FOTO 421: CASA DA VILA OPERÁRIA - ESTAÇÃO FERROVIÁRIA DE ITARARÉ. 307

FOTO 422: CASA DA VILA OPERÁRIA - ESTAÇÃO FERROVIÁRIA DE ITARARÉ 307

FOTO 423: CASA DA VILA OPERÁRIA - ESTAÇÃO FERROVIÁRIA DE ITARARÉ. .307

FOTO 424: CASA DA VILA OPERÁRIA - ESTAÇÃO FERROVIÁRIA DE ITARARÉ 307 
LISTA DE FIGURAS

FIGURA 1- PRANCHA DE J. B. DEBRET SOROCABA.

FIGURA 2- PRANCHA DE J. B. DEBRET SOBRE O RIO ITARARÉ. .37

FIGURA 3- PRANCHA DE J. B. DEBRET PASSAGEM POR CASTRO .38

FIGURA 4- PRANCHA DE J. B. DEBRET PASSAGEM PELOS CAMPOS GERAIS NO PARANÁ .38

FIGURA 5- JORNAL “O ITARARÉ” EM 1937 .52

FIGURA 6- AQUARELA DO SACO DE BATALHA EM 31/07/1932 POR ALFREDO FEIJÓ. .78

FIGURA 7- PRACHA DE J. B. DEBRET SOBRE ITAPEVA, A LONGA DISTÂNCIA.................86 FIGURA 8- PRACHA DE J. B. DEBRET SOBRE ITAPEVA, À CURTA DISTÂNCIA.................86 FIGURA 9- DESENHO DE ALENCAR ARARIPE EM 1887 SOBRE AS ISNCRIÇÕES DE FAXINA.

FIGURA 10- DESENHO DE DESIDÉRIO AYTAI SOBRE O ABRIGO ITAPEVA EM 1970

FIGURA 11- DESENHO DE ANDRÉ PROUS SOBRE O ABRIGO ITAPEVA EM 1979

FIGURA 12- DESENHO DE UM CERVÍDEO REALIZADO POR ANDRÉ PROUS EM 1979.....93

FIGURA 13: DESENHO DAS INSCRIÇÕES RUPESTRES DO ABRIGO DA FAZENDA POUSO ALTO DE BORDA REALIZADO POR SILVIO A.C. ARAÚJO EM 2006.

FIGURA 14: DESENHO DE VASILHAS ENCONTADAS POR PEREIRA JR. EM 1964 NO BAIRRO DA CAPUTERA EM ITAPEVA-SP

FIGURA 15: DESENHO DA PLANTA DA ESTAÇÃO FERROVIÁRIA DE FAXINA REALIZADA POR RAMOS DE AZEVEDO, VISTA LATERAL

FIGURA 16: DESENHO DA PLANTA DA ESTAÇÃO FERROVIÁRIA DE FAXINA REALIZADA POR RAMOS DE AZEVEDO, VISTA FRONATAL

FIGURA 17: DESENHO ESQUEMÁTICO DE UM ENCANADO TIPO ILHA FLUVIAL 225

FIGURA 18: GRAVURA DE J. B. DEBRET "ÍNDIOS SOLDADOS ESCOLTADO MULHERES E CRIANÇAS ÍNDIAS"

FIGURA 19: RECONSTITUIÇÃO VIRTUAL DE VASILHA CERÂMICA CORRUGADA, SÍTIO SANTA MARIA

FIGURA 20: RECONSTITUIÇÃO VISRTUAL DE VASILHA CERÂMICA CABOCLA ESCOVADA COM ALÇA, SÍTIO SANTA MARIA.

FIGURA 21: RECONSTITUIĈ̃̃O VIRTUAL DE UMA MORINGA COM APLIQUE PRÓXIMO A BORDA, SÍTIO SANTA MARIA.

FIGURA 22: RECONSTITUIÇÃO VIRTUAL DE PERFIL DE VASO CERÂMICO CORRUGADO COM ALÇA E ROLETADO COM DUPLAS INCISÕES PRÓXIMO A BORDA, SÍTIO HCR 
FIGURA 23: RECONSTITUIÇÃO VIRTUAL DE VASILHA CERÂMICA CORRUGADA E LISA PRÓXIMA A BASE, SÍTIO HCR.

FIGURA 24: RECONSTITUIÇÃO VIRTUAL DE PERFIL DE VASILHA CERÂMICA

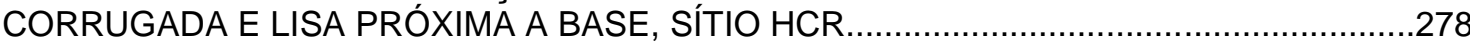

FIGURA 25: RECONSTITUIÇÃO VIRTUAL DE VASILHA CERÂMICA CORRUGADA, SÍTIO HCR

FIGURA 26: RECONSTITUIÇÃO VIRTUAL DE VASILHA CERÂMICA ESCOVADA E CARIMBADA NO FUNDO, SÍTIO HCR

FIGURA 27: RECONSTITUIÇÃO VISRTUAL DE PEQUENA MORINGA CERÂMICA COM

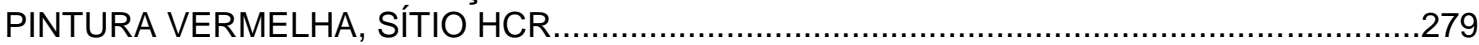

FIGURA 28: RECONSTITUIÇÃO VIRTUAL DE PEQUENA VASILHA CERÂMICALISA, SÍTIO HCR.

FIGURA 29: RECONSTITUIÇÃO VIRTUAL DE VASILHA CERÂMICA

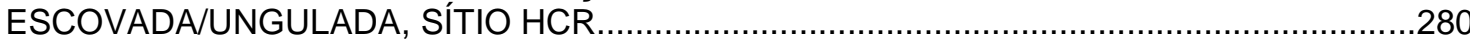

FIGURA 30: RECONSTITUIÇÃO VIRTUAL DE VASILHA CERÂMICA COM INCISOS SOB OS LÁBIOS, SÍTIO HCR.

FIGURA 31: RECONSTITUIÇÃO VIRTUAL DE PRATO CERÂMICO MARROM, SÍTIO HCR. 
LISTA DE TABELAS

TABELA 1: ESTRADA DE FERRO SOROCABANA ANOS DE INAUGURAÇÃO DA LINHA E CONSTRUÇÃO DAS ESTAÇÕES.

TABELA 2: DATAÇÕES DO SÍTIO FONSECA REALIZADAS POR PALLESTRINI EM 1970.

TABELA 3: DATAÇÕES DOS QUATRO NÍVEIS DO SÍTIO BRITAM (1983). .100

TABELA 4: DATAÇÕS DE CASAS SUBTERRÂNEAS REALIZADAS POR KAMASE EM 2004.

TABELA 5: SÍTIOS ARQUEOLÓGICOS IDENTIFICADOS E GEORREFERENCIADOS POR ARAÚJO (2001). ÁREA PILOTO TQG.

TABELA 6: SÍTIOS ARQUEOLÓGICOS IDENTIFICADOS E GEORREFERENCIADOS POR ARAÚJO (2001). ÁREA PILOTO CRC.

TABELA 7: SÍTIOS ARQUEOLÓGICOS IDENTIFICADOS E GEORREFERENCIADOS POR ARAÚJO (2001). ÁREA PILOTO RFD.

TABELA 8: SÍTIOS ARQUEOLÓGICOS IDENTIFICADOS E GEORREFERENCIADOS POR ARAÚJO (2001). ÁREA PILOTO BRR.

TABELA 9: SÍTIOS ARQUEOLÓGICOS IDENTIFICADOS E GEORREFERENCIADOS POR ARAÚJO (2001). ÁREA PILOTO TQS.

108

TABELA 10: SÍTIOS ARQUEOLÓGICOS IDENTIFICADOS E GEORREFERENCIADOS POR ARAÚJO (2001). ÁREA PLIOTO CDF.

TABELA 11: SÍTIOS ARQUEOLÓGICOS IDENTIFICADOS E GEORREFERENCIADOS POR ARAÚJO (2001). ÁREA PILOTO CRA. 108

TABELA 12: SÍTIOS ARQUEOLÓGICOS IDENTIFICADOS E GEORREFERENCIADOS POR

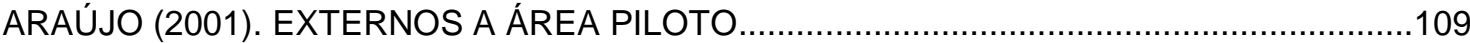

TABELA 13: DATAÇÕES ITARARÉ-TAQUARA REALIZADAS POR ARAÚJO (2001) ............110

TABELA 14: NOME E SIGLAS DOS SÍTIOS APONTADOS POR CAMARGO ARAÚJO EM 2006.

TABELA 15: COORDENADAS UTM DA ÁREA PILOTO BARRO BRANCO DE ACORDO COM SILVA-MÉNDES (2007)

TABELA 16: COORDENADAS UTM DA ÁREA PILOTO LIMEIRA DE ACORDO COM SILVAMÉNDES (2007) .

TABELA 17: COORDENADAS UTM DA ÁREA PILOTO LEITERIA DE ACORDO COM SILVAMÉNDES (2007).

TABELA 18: COORDENADAS UTM E DA ÁREA PILOTO CACHOEIRA DE ACORDO COM SILVA-MÉNDES (2007)

TABELA 19: COORDENADAS UTM DA ÁREA PILOTO LAGOA DE CIMA DE ACORDO COM SILVA-MÉNDES (2007)

TABELA 20: COORDENADAS UTM E DA ÁREA PILOTO OURO FINO DE ACORDO COM SILVA-MÉNDES (2007) 
TABELA 21: COORDENADAS UTM DA ÁREA PILOTO BOITUVA DE ACORDO COM SILVAMÉNDES (2007)

TABELA 22: COORDENADAS UTM DA ÁREA PILOTO BARREIRO DE ACORDO COM SILVA-MÉNDES (2007)

TABELA 23: COORDENADAS UTM DA ÁREA PILOTO INTERMONTES DE ACORDO COM SILVA-MÉNDES (2007)

TABELA 24: COORDENADAS UTM DA ÁREA PILOTO SÍTIO VELHO DE ACORDO COM SILVA-MÉNDES (2007)

TABELA 25: COORDENADAS UTM DA ÁREA PILOTO CRISTAL DE ACORDO COM SILVAMÉNDES (2007)

TABELA 26: COORDENADAS UTM DA ÁREA PILOTO ANACLETOS-VILLICOS DE ACORDO COM SILVA-MÉNDES (2007) 116

TABELA 27: COORDENADAS UTM DA ÁREA PILOTO TRIBOS-CAETANO DE ACORDO COM SILVA-MÉNDES (2007)

TABELA 28: SÍTIOS ARQUEOLÓGICOS DO PARANAPANEMA SUPERIOR PAULISTA FRUTOS DA IMPLANTAÇÃO DO GASBOL (DE BLASIS, 2000)

TABELA 29: SÍTIOS ARQUEOLÓGICOS DO PARANAPANEMA SUPERIOR PAULISTA FRUTOS DA IMPLANTAÇÃO IVAIPORÃ/ITABERÀ.

TABELA 30: SÍTIOS ARQUEOLÓGICOS DO PARANAPANEMA SUPERIOR PAULISTA FRUTOS DA IMPLANTAÇÃO IVAIPORÃ/ITABERÁ/TIJUCO PRETO

TABELA 31: SÍTIOS RESULTANTES DO PROGRAMA DE DIAGNÓSTICO ARQUEOLÓGICO DAS MINAS SP 01 E SP 04. 123

TABELA 32: COORDENADAS UTM DOS SÍTIOS ARQUEOLÓGICOS HISTÓRICOS

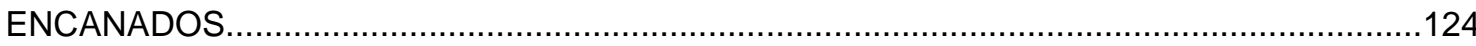

TABELA 33: COOREDANADAS UTM DAS OCORRÊNCIAS ARQUEOLÓGICAS DA AVALIAÇÃO DA FILITO/MAGNESITA S.A. 124

TABELA 34: SÍTIOS ARQUOLÓGICOS IDENTIFICADOS NO ESTUDO DO PATRIMÔNIO ARQUEOLÓGICO, ARQUITETÔNICO E PAISAGÍSTICO DA LT 500 KVA BATÉIAS/IBIÚNA 125

TABELA 35: SÍTIOS ARQUEOLÓGICOS IDENTIFICADOS NA AMPLIAÇÃO DA MINA CALCÁRIA LIMEIRA. 127

TABELA 36: PONTOS FORTES E PONTOS FRACOS DOS ENCANADOS 130

TABELA 37: COORDENADAS UTM DA OCORRÊNCIA ARQUEOLÓGICA DO DIAGGNÓSTICO DA MINERAÇÃO CRISTO REI. 131

TABELA 38: COOREDENADAS UTM DO SÍTIO ARQUEOLÓGICO HISTÓRICO PIÇARRÃO. 132

TABELA 39: COORDENADAS UTM DO SÍTIO LÍTICO DO ATERRO SANITÁRIO DE ITAPETININGA - ASI (CAMARGO ARAÚJO, 2008) 133

TABELA 40: COORDENADA UTM DO SÍTIO LÍTICO DA ESTAÇÃO DE TRATAMENTO DE ESGOSTO DE GUAREÍ. 
TABELA 41: RELAÇÃO TEMPO DE MAGISTÉRIO E CONHECIMENTO DE PRINCÍPIOS DE

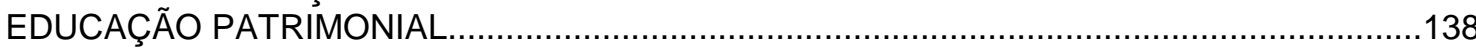

TABELA 42: QUADRO RESUMO DOS CENÁRIOS DE OCUPAÇÃO HUMANA PARA A ÁREA DE ESTUDO... 